\title{
On the Relationship Between Hierarchy and Type Morphisms*
}

\author{
Amanda Friedenberg ${ }^{\dagger} \quad$ Martin Meier $^{\ddagger}$
}

First Draft: October 2008

Current Draft: September 2009

\begin{abstract}
Here, we establish a relationship between hierarchy and type morphisms. Both concepts have been used to relate the types in one structure to types in a second "larger" structure. In general, the two concepts may differ, in the sense that a hierarchy morphism need not be a type morphism. We provide a necessary and sufficient condition under which the two concepts coincide. We go on to provide situations under which this condition must be satisfied.
\end{abstract}

Keywords: Hierarchies of Beliefs, Type Structures, Epistemic Game Theory, Morphisms JEL Codes: C70, C80

\section{Introduction}

Hierarchies of beliefs are fundamental, both to the epistemic analysis of games and to games of incomplete information. In practice, we follow Harsanyi [11, 1967-1968] and model these hierarchies of beliefs by using a type structure.

Figure 1.1 is an example of a game of incomplete information. It is essentially Example 1 in Dekel-Fudenberg-Morris [8, 2007]. As suggested above, to analyze this game, we need to specify what Ann believes about which matrix (in Figure 1.1, the $\theta_{1}$-section or the $\theta_{2}$-section) will obtain. We append to the game a type structure. Here's one example: There is one type of Ann, viz. $t^{a}$,

\footnotetext{
*We thank Pierpaolo Battigalli, Adam Brandenburger, Ethan Bueno de Mesquita, Aviad Heifetz, Marciano Siniscalchi, and Elias Tsakas for helpful conversations. We also thank a referee for invaluable suggestions. morphism s-09-0609.rap

${ }^{\dagger}$ Department of Economics, W.P. Carey School of Business, amanda.friedenberg@asu.edu, www.public.asu.edu/ $\sim$ afrieden.

‡Institut für Höhere Studien, Martin.Meier@ihs.ac.at.
} 
and one type of Bob, viz. $t^{b}$. Type $t^{a}$ assigns probability $\frac{1}{2}$ to $\left(\theta_{1}, t^{b}\right)$ and probability $\frac{1}{2}$ to $\left(\theta_{2}, t^{b}\right)$. Likewise, $t^{b}$ assigns probability $\frac{1}{2}$ to $\left(\theta_{1}, t^{a}\right)$ and probability $\frac{1}{2}$ to $\left(\theta_{2}, t^{a}\right)$.
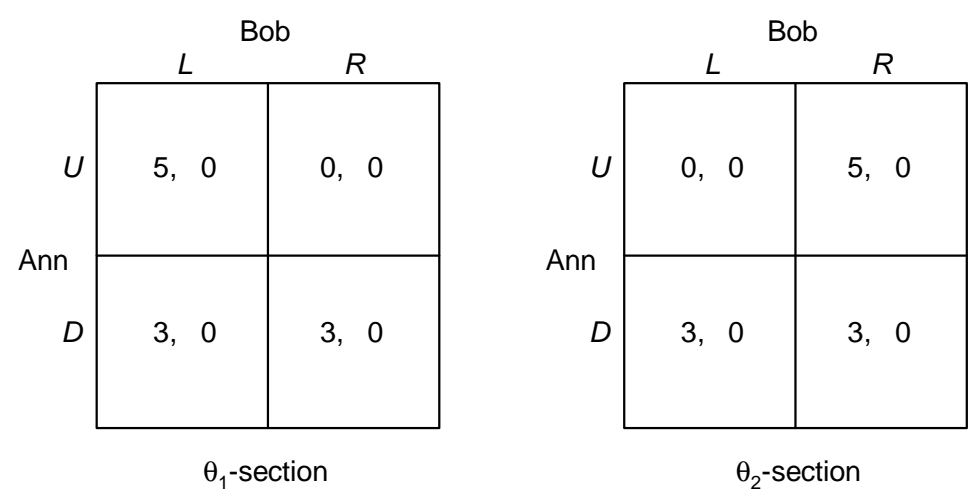

Figure 1.1

Here we focus on the question: When is one type structure "contained in" another type structure? The literature has considered two ideas. The first idea is that a type structure $\mathcal{T}$ is contained in a type structure $\mathcal{T}_{*}$ if every hierarchy of beliefs associated with a type in $\mathcal{T}$ is also associated with a type in $\mathcal{T}_{*}$. In this case, we say that there is a hierarchy morphism from $\mathcal{T}$ to $\mathcal{T}_{*}$. The second idea is that a type structure $\mathcal{T}$ is contained in $\mathcal{T}_{*}$, if we can "embed" $\mathcal{T}$ into $\mathcal{T}_{*}$ via a map called a type morphism. Type morphisms preserve the beliefs associated with a type structure, and do not make direct reference to hierarchies of beliefs.

Both hierarchy and type morphisms have a long tradition in the literature. The former goes back to Böge-Eisele [4, 1979] and the latter to Mertens-Zamir [17, 1985]. Hierarchy morphisms arise naturally in certain game-theoretic questions. Specifically, they appear in both the epistemic and robustness literatures. Type morphisms have played an instrumental role in constructing large type structures; this is made explicit in Heifetz-Samet [12, 1998]. They also have played a more subtle role in the robustness literature.

Back to our example. Take the structure $\mathcal{T}$ to be the one already studied. Consider a second type structure $\mathcal{T}_{*}$, where there is one type for Ann, viz. $t_{*}^{a}$, and there are two types for Bob viz. $t_{*}^{b}$ and $u_{*}^{b}$. Type $t_{*}^{a}$ assigns probability $\frac{1}{2}$ to $\left(\theta_{1}, t_{*}^{b}\right)$ and probability $\frac{1}{2}$ to $\left(\theta_{2}, u_{*}^{b}\right)$. Here, $t^{a}$ and $t_{*}^{a}$ (resp. $t^{b}, t_{*}^{b}$, and $u_{*}^{b}$ ) each induces the same hierarchies of beliefs-i.e., each assigns probability $\frac{1}{2}: \frac{1}{2}$ to $\theta_{1}: \theta_{2}$, each believes the other player assigns probability $\frac{1}{2}: \frac{1}{2}$ to $\theta_{1}: \theta_{2}$, etc. So, here, there is a hierarchy morphism from $\mathcal{T}$ to $\mathcal{T}_{*} \cdot 1$

But while there is a hierarchy morphism from $\mathcal{T}$ to $\mathcal{T}_{*}$, we will see that there is no type morphism

\footnotetext{
${ }^{1}$ There is also a hierarchy morphism from $\mathcal{T}_{*}$ to $\mathcal{T}$.
} 
from $\mathcal{T}$ to $\mathcal{I}_{*}$. And, this distinction is behaviorally relevant. In Figure 1.1, when we go from the type structure $\mathcal{T}$ to the type structure $\mathcal{T}_{*}$ (via the hierarchy morphism), we add a Bayesian equilibrium. To see this, begin with the structure $\mathcal{T}$ and note that, in any Bayesian equilibrium, $t^{a}$ plays Down with probability one. Turning to $\mathcal{T}_{*}$, there is a Bayesian equilibrium in which $t_{*}^{a}$ plays Down, $t_{*}^{b}$ plays Left, and $u_{*}^{b}$ plays Right. So, we add equilibrium predictions in going (under the hierarchy morphism) from $\mathcal{T}$ to $\mathcal{T}_{*}$. Contrast this with type morphisms: If there is a type morphism from $\mathcal{T}$ to $\mathcal{T}_{*}$, then we do not add an equilibrium prediction in going from $\mathcal{T}$ to $\mathcal{T}_{*}$. (See Proposition 4.1 in [9, 2009].)

This is essentially the leading example in Dekel-Fudenberg-Morris [8, 2007] and Ely-Peski [7, 2006]. They noted that a Bayesian equilibrium prediction need not be preserved under hierarchy morphisms. But, to the best of our knowledge, they did not note the distinction between hierarchy and type morphisms in such examples. In Section 9, we will come back and discuss this distinction at the conceptual level, with an eye toward connecting it to the idea of robustness.

The body of this paper addresses a formal question: What is the relationship between hierarchy and type morphisms? Heifetz-Samet [12, 1998] provide a first-cut answer. They show that every type morphism is a hierarchy morphism. We address the converse: When is every hierarchy morphism a type morphism?

To better understand the question and the approach we will take, think of the relationship between Harsanyi [11, 1967-1968] and Mertens-Zamir [17, 1985]. Harsanyi [11, 1967-1968] introduced the concept of a type structure and argued that it can model certain hierarchies of beliefs, in the sense that a type structure induces hierarchies of beliefs. But the fact that a type structure induces hierarchies of beliefs does not immediately imply that it can always provide an adequate model of hierarchies. For this we require more - we require that each hierarchy of beliefs can indeed be modelled as a type in some such structure. Mertens-Zamir [17, 1985] provided conditions under which this is the case.

Now turn to the question here. Can one structure be mapped into a second structure, in a way that preserves hierarchies of beliefs? The answer is yes if and only if there is a hierarchy morphism from the first structure into the second structure. But, by definition, this answer requires that we leave the purview of Harsanyi's [11, 1967-1968] type structure model. Type morphisms provide an alternate answer. They are defined entirely within the purview of the type structure model and map one structure into a second in a way that preserves hierarchies of beliefs. But they can only act as a model for hierarchy morphisms if every hierarchy morphism is indeed a type morphism.

We provide a necessary and sufficient condition for each hierarchy morphism to be a type morphism. Specifically, we show:

Result: Fix a type structure $\mathcal{T}_{*}$. Any hierarchy morphism from some structure $\mathcal{T}$ to $\mathcal{T}_{*}$ is also a type morphism from $\mathcal{T}$ to $\mathcal{T}_{*}$ if and only if the measurable sets in $\mathcal{T}_{*}$ satisfy a certain measurability condition, which we call strong measurability.

In Section 5, we formally define strong measurability. For now we note that it is a condition defined 
entirely within the domain of the type structure model - it does not make reference to hierarchies of beliefs. We go on to provide a substantive interpretation of this condition. We show that a type structure is strongly measurable if and only if the measurable sets are generated by the hierarchies of beliefs. So, strong measurability then says that a player can reason about an event in type space if and only if the event is induced by the player's hierarchy language. (Again, this is a result. Strong measurability itself is defined on the type structure alone.)

This raises the question: Are there (natural) assumptions under which a type structure must be strongly measurable? We provide one such set of assumptions. In particular, we show that under standard Borel and non-redundancy conditions, a type structure is indeed strongly measurable. Note, non-redundancy is the requirement that no two types induce the same hierarchies of beliefs. So, in the above example, the structure $\mathcal{T}_{*}$ is redundant - the types $t_{*}^{b}$ and $u_{*}^{b}$ induce the same hierarchies of beliefs. While non-redundancy is typically defined as a property of hierarchies of beliefs, we show that we need not leave the domain of type structures to check whether the condition holds. In particular, building on Proposition 2.5 in Mertens-Zamir [17, 1985], we provide a characterization of non-redundancy that is defined entirely within the type structure model.

Let us reiterate the motivation: Type structures are the predominant model of hierarchies of beliefs. The standard view in the literature is that type structures are operationally more convenient than working directly with hierarchies of beliefs. Moreover, often times, game-theoretic questions require that we study a particular type structure - one that is specifically different from the canonical construction. (This is true both in the literature on incomplete information and in the epistemic program ${ }_{2}^{2}$ But, certain game-theoretic questions also require that we talk about all mappings that preserve hierarchies of beliefs, i.e., from smaller structures to larger structures. (This is particularly true in the literature on the robustness to misspecifying the parameter set, e.g., Dekel-FudenbergMorris [8, 2007], Ely-Peski [7, 2006], Liu [15, 2009], Sadzik [20, 2009], etc. Such mappings also arise in other robustness questions, e.g., Friedenberg-Meier [9, 2007], Weinstein-Yildiz [22, 2007], Yildiz [24, 2009], etc., and in the epistemic game theory literature, e.g., Friedenberg [10, 2008].) In each of these papers, the game theoretic question naturally starts with hierarchy morphisms. There is the question of whether the analyst can instead start with type morphisms, i.e., whether the analyst can use type morphisms as a model for hierarchy morphisms. The results here speak to when the analyst can vs. cannot do so. In particular, the results give necessary and sufficient conditions to guarantee the analyst can use the type morphism model (and, these conditions do not leave the domain of the type structure model). Thus, in a sense, we do for structure preserving maps what Mertens-Zamir [17, 1985] did for type structures themselves.

How should this paper be viewed? This is one of many papers that add to our understanding of type structures and hierarchies of beliefs. (Some classics include Armbruster-Boge [2, 1979], Böge-Eisele [4, 1979], Mertens-Zamir [17, 1985], Bradenburger-Dekel [5, 1993], and Heifetz-Samet [12, 1998]-[13, 1999]. See Siniscalchi [21, 2008] for a recent overview.) That is, it is one of many

\footnotetext{
${ }^{2}$ In each of these literatures, the substantive application drives what type structure is studied — very much like the substantive application drives what game itself is studied.
} 
papers that contributes to the language of game theory. Much like other papers in this literature, we were inspired by the game theoretic application. But, as with other papers in this literature, we stop short of directly applying our results to get new implications for behavior. Instead, we view this as a first step, to be applied to get a full-fledged game-theoretic analysis.

We now turn to the formal treatment. Section 2 provides notation. Definitions of hierarchy and type morphisms are provided in Section 3. Section 4 gives two examples of hierarchy morphisms that are not type morphisms. These motivate the main result in Section 5 namely a necessary and sufficient condition for the two concepts to coincide. The proofs are given in Section 6 . In Section

7. we explore when the condition is or is not met. Section 8 discusses an alternate set of sufficient conditions. Finally, in Section 9 , we conclude by returning to the game theory application.

\section{Type Structures and Hierarchies of Beliefs}

Write $(\Omega, \mathcal{S}(\Omega))$ for a measurable space, where $\mathcal{S}(\Omega)$ is the sigma algebra on $\Omega$. Throughout, we endow the product of measurable spaces with the product sigma-algebra. Given a set $\Phi$, a measurable space $(\Omega, \mathcal{S}(\Omega)$ ), and a function $f: \Phi \rightarrow \Omega$, write $\sigma(f)$ for the sigma algebra on $\Phi$ generated by $f$, i.e., $F \in \sigma(f)$ if and only if there exists some $E \in \mathcal{S}(\Omega)$ with $F=f^{-1}(E)$.

Let $\Delta(\Omega, \mathcal{S}(\Omega))$ be the set of probability measures on $(\Omega, \mathcal{S}(\Omega))$. When it is clear from the context which sigma-algebra we are considering, we suppress reference to $\mathcal{S}(\Omega)$ and simply write $\Delta(\Omega)$. Throughout, we will endow the set $\Delta(\Omega, \mathcal{S}(\Omega))$ with the sigma-algebra generated by maps $\mu \mapsto \mu(E)$, i.e., for all $E \in \mathcal{S}(\Omega) !^{3}$ We will write $\mathcal{M}(\Delta(\Omega))$ to clarify that we are restricting attention to the sigma-algebra generated by these maps.

Note, $\mathcal{M}(\Delta(\Omega))$ corresponds to two standards in the literature. First, $\mathcal{M}(\Delta(\Omega))$ is the sigmaalgebra used in Heifetz-Samet [12, 1998]. In particular, $\mathcal{M}(\Delta(\Omega))$ is also generated by sets of the form $\{\mu \in \Delta(\Omega, \mathcal{S}(\Omega)): \mu(E) \geq p\}$, across all $E \in \mathcal{S}(\Omega)$ and $p \in[0,1]$. (Use Kechris [14, 1995; page 67] and the fact that the Borel sets on $[0,1]$ are generated by sets of the form $[p, 1]$.) Second, when $\Omega$ is metrizable and $\mathcal{S}(\Omega)$ is the Borel sigma-algebra, $\mathcal{M}(\Delta(\Omega))$ is the set of Borel sets on $\Delta(\Omega)$ generated by the weak topology. (Use Lemma 14.16 in Aliprantis-Border [1, 1999] and the first standard.)

Fix measurable spaces $(\Omega, \mathcal{S}(\Omega))$ and $(\Phi, \mathcal{S}(\Phi))$. Consider the associated measurable spaces $(\Delta(\Omega), \mathcal{M}(\Delta(\Omega)))$ and $(\Delta(\Phi), \mathcal{M}(\Delta(\Phi)))$. Given a measurable map $f: \Omega \rightarrow \Phi$, write $\underline{f}: \Delta(\Omega) \rightarrow$ $\Delta(\Phi)$ for the map where $\underline{f}(\mu)$ is the image measure of $\mu$ under $f$. Note the map $\underline{f}$ is also measurable 4

We consider a two player situation, with players $a$ and $b$. (The generalization to three or more players is immediate.) There is a parameter set $\Theta$, which reflects the players' uncertainty. (In the example of Figure 1.1, $\Theta=\left\{\theta_{1}, \theta_{2}\right\}$ and the realization of $\Theta$ determined the players' payoff

\footnotetext{
${ }^{3}$ Note, we endow the real numbers with the usual Borel $\sigma$-algebra.

${ }^{4}$ Use two facts. First, $\mathcal{M}(\Delta(\Phi))$ is generated by sets of the form $\{\mu \in \Delta(\Phi): \mu(E) \geq p\}$, and likewise $\mathcal{M}(\Delta(\Omega))$. Second, each set $\underline{f}^{-1}(\{\mu \in \Delta(\Phi): \mu(E) \geq p\})$ can be written as $\left\{\nu \in \Delta(\Omega): \nu\left(f^{-1}(E)\right) \geq p\right\}$.
} 
matrix.) The implicit assumption is that the parameter set $\Theta$ is "transparent" to the players, but the realization of $\Theta$ is not known to the players. As such, they each have a hierarchy of belief based on the parameter set $\Theta$, i.e., they each have a belief about (the realization of) $\Theta$, they each have a belief about " $\Theta$ and the other player's belief about $\Theta$," and so on. Following Harsanyi 11, 1967-1968], we model such hierarchies via a type structure.

A type structure is based on a parameter space $(\Theta, \mathcal{S}(\Theta))$, i.e., a parameter set $\Theta$ and an associated sigma algebra $\mathcal{S}(\Theta)$. The sets in $\mathcal{S}(\Theta)$ reflect the subsets of $\Theta$ that the players can reason about, i.e., it reflects the players' language about $\Theta$.

Definition 2.1 An $(\Theta, \mathcal{S}(\Theta))$-based type structure is some

$$
\mathcal{T}=\left\langle(\Theta, \mathcal{S}(\Theta)) ;\left(T^{a}, \mathcal{S}\left(T^{a}\right)\right),\left(T^{b}, \mathcal{S}\left(T^{b}\right)\right) ; \beta^{a}, \beta^{b}\right\rangle
$$

where $\beta^{a}, \beta^{b}$ are measurable maps with $\beta^{a}: T^{a} \rightarrow \Delta\left(\Theta \times T^{b}\right)$ and $\beta^{b}: T^{b} \rightarrow \Delta\left(\Theta \times T^{a}\right)$. We call $T^{a}, T^{b}$ type sets and we call $\beta^{a}, \beta^{b}$ belief maps.

Indeed, $(\Theta, \mathcal{S}(\Theta))$-based type structure induces hierarchies of beliefs about the parameters of the game. To see this, begin with the measurable space $\left(Z_{1}, \mathcal{S}\left(Z_{1}\right)\right)=(\Theta, \mathcal{S}(\Theta))$. Inductively define $Z_{m+1}=Z_{m} \times \Delta\left(Z_{m}\right)$. Define maps $\rho_{m}^{a}: \Theta \times T^{b} \rightarrow Z_{m}$ and $\rho_{m}^{b}: \Theta \times T^{a} \rightarrow Z_{m}$ as follows: Let

$$
\begin{aligned}
\rho_{1}^{a}\left(\theta, t^{b}\right) & =\theta \\
\rho_{m+1}^{a}\left(\theta, t^{b}\right) & =\left(\rho_{m}^{a}\left(\theta, t^{b}\right), \underline{\rho}_{m}^{b}\left(\beta^{b}\left(t^{b}\right)\right)\right),
\end{aligned}
$$

and similarly with $a$ and $b$ interchanged. Then, define $\delta_{m}^{a}: T^{a} \rightarrow \Delta\left(Z_{m}\right)$ so that $\delta_{m}^{a}=\underline{\rho}_{m}^{a} \circ \beta^{a}$. A standard induction argument gives that, for each $m$, the maps $\rho_{m}^{a}, \underline{\rho}_{m}^{a}$, and $\delta_{m}^{a}$ are measurable. With this $\delta^{a}: T^{a} \rightarrow \prod_{m=1}^{\infty} \Delta\left(Z_{m}\right)$ will specify the hierarchy induced by each type, i.e., $\delta^{a}\left(t^{a}\right)=\left(\delta_{1}^{a}\left(t^{a}\right), \delta_{2}^{a}\left(t^{a}\right), \ldots\right)$. And similarly with $a$ and $b$ interchanged. Since each of the maps $\delta_{m}^{a}$ and $\delta_{m}^{b}$ are measurable, the maps $\delta^{a}$ and $\delta^{b}$ are also measurable.

We point out, in passing: A $(\Theta, \mathcal{S}(\Theta)$ )-based type structure represents a set of restrictions on players' hierarchies of beliefs that are "transparent" to the players. (See [3, 2009; Appendix A.II] for a formal equivalence result.)

\section{Hierarchy and Type Morphisms}

In what follows below, we will fix two $(\Theta, \mathcal{S}(\Theta))$-based structures

$$
\mathcal{T}=\left\langle(\Theta, \mathcal{S}(\Theta)) ;\left(T^{a}, \mathcal{S}\left(T^{a}\right)\right),\left(T^{b}, \mathcal{S}\left(T^{b}\right)\right) ; \beta^{a}, \beta^{b}\right\rangle
$$

and

$$
\mathcal{T}_{*}=\left\langle(\Theta, \mathcal{S}(\Theta)) ;\left(T_{*}^{a}, \mathcal{S}\left(T_{*}^{a}\right)\right),\left(T_{*}^{b}, \mathcal{S}\left(T_{*}^{b}\right)\right) ; \beta_{*}^{a}, \beta_{*}^{b}\right\rangle .
$$


We will write $\rho_{m}^{a}, \delta_{m}^{a}$, and $\delta^{a}$ for the maps associated with $\mathcal{T}$ and $\rho_{m, *}^{a}, \delta_{m, *}^{a}$, and $\delta_{*}^{a}$ for the maps associated with $\mathcal{T}_{*}$.

Definition 3.1 Let $h^{a}: T^{a} \rightarrow T_{*}^{a}$ and $h^{b}: T^{b} \rightarrow T_{*}^{b}$ be maps so that $\delta_{*}^{a} \circ h^{a}=\delta^{a}$ and $\delta_{*}^{b} \circ h^{b}=$ $\delta^{b}$. Then, $h^{a}$ and $h^{b}$ are hierarchy morphisms (from $\mathcal{T}$ to $\mathcal{T}_{*}$ ). In this case, we will abuse terminology and say the pair $\left(h^{a}, h^{b}\right)$ is a hierarchy morphism (from $\mathcal{T}$ to $\mathcal{T}_{*}$ ).

We point out:

Remark 3.1 There is a hierarchy morphism from $\mathcal{T}$ to $\mathcal{T}_{*}$ if and only if $\delta^{a}\left(T^{a}\right) \subseteq \delta_{*}^{a}\left(T_{*}^{a}\right)$ and $\delta^{b}\left(T^{b}\right) \subseteq \delta_{*}^{b}\left(T_{*}^{b}\right)$.

Now we turn to type morphisms. Given maps $f_{1}: \Omega_{1} \rightarrow \Phi_{1}$ and $f_{2}: \Omega_{2} \rightarrow \Phi_{2}$, we write $f_{1} \times f_{2}$ for the map from $\Omega_{1} \times \Omega_{2}$ to $\Phi_{1} \times \Phi_{2}$ so that $\left(f_{1} \times f_{2}\right)\left(\omega_{1}, \omega_{2}\right)=\left(f_{1}\left(\omega_{1}\right), f_{2}\left(\omega_{2}\right)\right)$. We also write id : $\Theta \rightarrow \Theta$ for the identity map.

Definition 3.2 (Mertens-Zamir [17, 1985]) Let $h^{a}: T^{a} \rightarrow T_{*}^{a}$ and $h^{b}: T^{b} \rightarrow T_{*}^{b}$ be measurable maps, so that $\left(\underline{\mathrm{id} \times h^{b}}\right) \circ \beta^{a}=\beta_{*}^{a} \circ h^{a}$ and $\left(\underline{\mathrm{id} \times h^{a}}\right) \circ \beta^{b}=\beta_{*}^{b} \circ h^{b}$. Then $\left(h^{a}, h^{b}\right)$ is called a type morphism (from $\mathcal{T}$ to $\mathcal{T}_{*}$ ).

(Note, in Definition 3.2, we cannot drop the requirement that $h^{a}$ and $h^{b}$ are measurable. It is required for the maps $\underline{\mathrm{id} \times h^{a}}$ and $\underline{\mathrm{id} \times h^{b}}$ to be well-defined.) Definition 3.2 can be illustrated in Figure 2.1: A type morphism, viz. $\left(h^{a}, h^{b}\right)$, requires that the following diagram commutes.

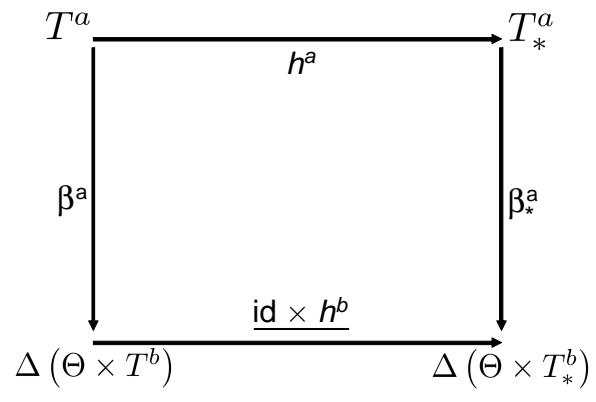

Figure 2.1

Thus, in a certain sense, a type morphism asks for beliefs to be preserved. Indeed, Heifetz-Samet [12, 1998] tell us that we can go further - a type morphism preserves hierarchies of beliefs.

Proposition 3.1 (Heifetz-Samet [12, 1998; Proposition 5.1]) If $\left(h^{a}, h^{b}\right)$ is a type morphism from $\mathcal{T}$ to $\mathcal{T}_{*}$, then $\left(h^{a}, h^{b}\right)$ is also a hierarchy morphism.

(Heifetz-Samet [12, 1998] define hierarchies of beliefs somewhat differently than here. That said, their proof can be replicated in this formalism.) 


\section{Hierarchy Morphisms May Not Be Type Morphisms}

In Section 11 we saw that a hierarchy morphism, viz. $\left(h^{a}, h^{b}\right)$, need not be a type morphism. Now we will see that there are two potential reasons for this failure. First, a hierarchy morphism need not be measurable. Second, even if it is measurable, it may fail to preserve the belief maps, e.g., it may fail the requirement that $\left(\underline{\mathrm{id} \times h^{b}}\right) \circ \beta^{a}=\beta_{*}^{a} \circ h^{a}$. Let us first see examples of each of these phenomena.

Example 4.1 This is an example of a hierarchy morphism that is not measurable, and so not a type morphism.

Take $\Theta=[0,1]$. Endow $\Theta$ with the usual topology and take $\mathcal{S}(\Theta)$ to be the set of Borel sets on $\Theta$. We will consider two $(\Theta, \mathcal{S}(\Theta))$-based structures

$$
\mathcal{T}=\left\langle(\Theta, \mathcal{S}(\Theta)) ;\left(T^{a}, \mathcal{S}\left(T^{a}\right)\right),\left(T^{b}, \mathcal{S}\left(T^{b}\right)\right) ; \beta^{a}, \beta^{b}\right\rangle
$$

and

$$
\mathcal{T}_{*}=\left\langle(\Theta, \mathcal{S}(\Theta)) ;\left(T_{*}^{a}, \mathcal{S}\left(T_{*}^{a}\right)\right),\left(T_{*}^{b}, \mathcal{S}\left(T_{*}^{b}\right)\right) ; \beta_{*}^{a}, \beta_{*}^{b}\right\rangle .
$$

Both structures have the same type sets-specifically, $T^{a}=T_{*}^{a}=[0,1]$ and $T^{b}=T_{*}^{b}=\left\{t^{b}\right\}$. However, we take $\mathcal{S}\left(T^{a}\right)=\mathcal{S}(\Theta)$ and $\mathcal{S}\left(T_{*}^{a}\right)=2^{[0,1]}$. Choose the maps $\beta^{a}$ and $\beta_{*}^{a}$ so that, for each $t^{a} \in[0,1]$,

$$
\beta^{a}\left(t^{a}\right)\left(\left\{\left(t^{a}, t^{b}\right)\right\}\right)=\beta_{*}^{a}\left(t^{a}\right)\left(\left\{\left(t^{a}, t^{b}\right)\right\}\right)=1 .
$$

Likewise, choose the maps $\beta^{b}$ and $\beta_{*}^{b}$ so that

$$
\beta^{b}\left(t^{b}\right)(\{(0,0)\})=\beta_{*}^{b}\left(t^{b}\right)(\{(0,0)\})=1 .
$$

Now, consider the pair $\left(h^{a}, h^{b}\right)$, where $h^{a}: T^{a} \rightarrow T_{*}^{a}$ and $h^{b}: T^{b} \rightarrow T_{*}^{b}$ are the identity maps. It is readily verified that this is a hierarchy morphism. But this is not a type morphism. To see this, note that we can find some $E_{*} \subseteq[0,1]$ that is not Lebesgue measurable (see Oxtoby [18, Theorem 5.5; 1980]) and any such set is not contained in $\mathcal{S}\left(T^{a}\right)$ (see Oxtoby [18, Theorem 3.16; 1980]). We have that $E_{*} \in \mathcal{S}\left(T_{*}^{a}\right)$, but $E_{*}=\left(h^{a}\right)^{-1}\left(E_{*}\right) \notin \mathcal{S}\left(T^{a}\right)$, so that $h^{a}$ is not measurable. As such, $\left(h^{a}, h^{b}\right)$ cannot be a type morphism. Indeed, there is no type morphism from $\mathcal{T}$ to $\mathcal{T}_{*}$.

Example 4.2 This is an example of a measurable hierarchy morphism that is not a type morphism. It is essentially the example in Section 1 .

Take $\Theta=\left\{\theta_{1}, \theta_{1}\right\}$ and take $\mathcal{S}(\Theta)=2^{\Theta}$. We will consider two $(\Theta, \mathcal{S}(\Theta))$-based structures,

$$
\mathcal{T}=\left\langle(\Theta, \mathcal{S}(\Theta)) ;\left(T^{a}, \mathcal{S}\left(T^{a}\right)\right),\left(T^{b}, \mathcal{S}\left(T^{b}\right)\right) ; \beta^{a}, \beta^{b}\right\rangle
$$

and

$$
\mathcal{T}_{*}=\left\langle(\Theta, \mathcal{S}(\Theta)) ;\left(T_{*}^{a}, \mathcal{S}\left(T_{*}^{a}\right)\right),\left(T_{*}^{b}, \mathcal{S}\left(T_{*}^{b}\right)\right) ; \beta_{*}^{a}, \beta_{*}^{b}\right\rangle .
$$


Specifically, for the structure $\mathcal{T}$, take $T^{a}=\left\{t^{a}\right\}$ and $T^{b}=\left\{t^{b}\right\}$. Then, set

$$
\begin{aligned}
& \beta^{a}\left(t^{a}\right)\left(\left\{\left(\theta_{1}, t^{b}\right)\right\}\right)=\beta^{a}\left(t^{a}\right)\left(\left\{\left(\theta_{2}, t^{b}\right)\right\}\right)=\frac{1}{2} \\
& \beta^{b}\left(t^{b}\right)\left(\left\{\left(\theta_{1}, t^{a}\right)\right\}\right)=\beta^{b}\left(t^{b}\right)\left(\left\{\left(\theta_{2}, t^{a}\right)\right\}\right)=\frac{1}{2} .
\end{aligned}
$$

For the structure $\mathcal{T}_{*}$, take $T_{*}^{a}=\left\{t_{*}^{a}\right\}, T_{*}^{b}=\left\{t_{*}^{b}, u_{*}^{b}\right\}$, and let $\mathcal{S}\left(T_{*}^{b}\right)$ be the power set of $T_{*}^{b}$. Then, set

$$
\begin{aligned}
& \beta_{*}^{a}\left(t_{*}^{a}\right)\left(\left\{\left(\theta_{1}, t_{*}^{b}\right)\right\}\right)=\beta_{*}^{a}\left(t_{*}^{a}\right)\left(\left\{\left(\theta_{2}, u_{*}^{b}\right)\right\}\right)=\frac{1}{2} \\
& \beta_{*}^{b}\left(t_{*}^{b}\right)\left(\left\{\left(\theta_{1}, t_{*}^{a}\right)\right\}\right)=\beta_{*}^{b}\left(t_{*}^{b}\right)\left(\left\{\left(\theta_{2}, t_{*}^{a}\right)\right\}\right)=\frac{1}{2} \\
& \beta_{*}^{b}\left(u_{*}^{b}\right)\left(\left\{\left(\theta_{1}, t_{*}^{a}\right)\right\}\right)=\beta_{*}^{b}\left(u_{*}^{b}\right)\left(\left\{\left(\theta_{2}, t_{*}^{a}\right)\right\}\right)=\frac{1}{2} .
\end{aligned}
$$

Consider the maps $\left(h^{a}, h^{b}\right)$ with $h^{a}\left(t^{a}\right)=t_{*}^{a}$ and $h^{b}\left(t^{b}\right)=t_{*}^{b}$. It is readily verified that this is a measurable hierarchy morphism. But $\beta_{*}^{a}\left(h^{a}\left(t^{a}\right)\right)\left(\left\{\left(\theta_{2}, u_{*}^{b}\right)\right\}\right)=\frac{1}{2}$ and $\beta^{a}\left(t^{a}\right)\left(\left\{\theta_{2}\right\} \times\right.$ $\left.\left(\left(h^{b}\right)^{-1}\left(\left\{u_{*}^{b}\right\}\right)\right)\right)=\beta^{a}\left(t^{a}\right)(\emptyset)=0$. This says that $\left(h^{a}, h^{b}\right)$ is not a type morphism. Indeed, there is no type morphism from $\mathcal{T}$ to $\mathcal{T}_{*}$.

We have seen two problems - a hierarchy morphism may fail to be measurable and, even if it is measurable, it may still fail to preserve the belief maps.

First, note that, for a hierarchy morphism (from $\mathcal{T}$ to $\mathcal{T}_{*}$ ) to be measurable, we want to impose restrictions on the measurable sets in $\mathcal{S}\left(T_{*}^{a}\right)$ and $\mathcal{S}\left(T_{*}^{b}\right)$. (It will be easier to satisfy the measurability condition, if there are fewer measurable sets.) Suppose we consider the smallest sigma-algebra on $T_{*}^{a}$ so that the hierarchies map is measurable $5^{5}$ Then, any measurable set in $T_{*}^{a}$ is induced by a measurable set of hierarchies. With this, we can use the fact that the map from $T^{a}$ to hierarchies is measurable, and get that our hierarchy morphism must also be measurable.

Now turn to Example 4.2. There, the hierarchy morphism was measurable, despite the fact that there are measurable sets in $T_{*}^{b}$ that are not generated by hierarchies. (Specifically, the events $\left\{t_{*}^{b}\right\}$ and $\left\{u_{*}^{b}\right\}$ are not generated by hierarchies.) Indeed, this is important for the failure of the hierarchy morphism to preserve belief maps. To see this, note that $h^{a}\left(t^{a}\right)$ assigns probability one to $u_{*}^{b}$, despite the fact that $t^{a}$ assigns probability one to $t^{b}$ and $h^{b}\left(t^{b}\right) \neq u_{*}^{b}$. Thus, if we are to preserve hierarchies of beliefs, then $h^{b}\left(t^{b}\right)$ and $u_{*}^{b}$ must be associated with the same hierarchies of beliefs. But, then, the fact that these two types are in different measurable sets (in $T_{*}^{b}$ ) tells us that the measurable structure of $T_{*}^{b}$ is not generated by the hierarchies map.

This fact will be true more generally: If the measurable structure of $T_{*}^{a}$ and $T_{*}^{b}$ is generated by the measurable structure on hierarchies, then any hierarchy preserving map is also a belief preserving map. Thus, we have one solution for the two problems. Moreover, we will also see a converse: If

\footnotetext{
${ }^{5}$ Note: We could not change the measurable structure and dispense with the property that the hierarchies map is measurable. This property is essential for hierarchies of beliefs to be well-defined.
} 
the measurable structure of $T_{*}^{a}$ or $T_{*}^{b}$ is not generated by the measurable structure on hierarchies, then we can always find some hierarchy morphism that is not a type morphism.

That said, the condition that the measurable structure of $T_{*}^{a}$ and $T_{*}^{b}$ is generated by the measurable structure on hierarchies requires explicitly specifying hierarchies of beliefs. Put differently, it requires leaving the purview of the type structure model. We begin with an equivalent formalization defined on the type structure alone.

\section{When Hierarchy Morphisms are Type Morphisms}

We begin with the measurability condition, which is due to Mertens-Zamir [17, page 6; 1985].

Definition 5.1 Fix a $(\Theta, \mathcal{S}(\Theta))$-based structure $\mathcal{T}$ and subsigma algebras $\mathcal{F}_{i}\left(T^{a}\right) \subseteq \mathcal{S}\left(T^{a}\right)$ and $\mathcal{F}_{i}\left(T^{b}\right) \subseteq \mathcal{S}\left(T^{b}\right)$. Say $\mathcal{F}_{i}\left(T^{a}\right) \times \mathcal{F}_{i}\left(T^{b}\right)$ is closed under $\mathcal{T}$ if, for each $E \times F^{b} \in \mathcal{S}(\Theta) \times \mathcal{F}_{i}\left(T^{b}\right)$ and each $p \in[0,1]$,

$$
\left\{t^{a} \in T^{a}: \beta^{a}\left(t^{a}\right)\left(E \times F^{b}\right) \geq p\right\} \in \mathcal{F}_{i}\left(T^{a}\right),
$$

and, likewise, for $b$.

Fix a $(\Theta, \mathcal{S}(\Theta))$-based structure $\mathcal{T}$. Let $\left\{\mathcal{F}_{i}\left(T^{a}\right) \times \mathcal{F}_{i}\left(T^{b}\right)\right\}$ be the collection of all subsigmaalgebras that are closed under $\mathcal{T}$. Define $\mathcal{F}\left(T^{a}\right)=\bigcap_{i} \mathcal{F}_{i}\left(T^{a}\right)$ and $\mathcal{F}\left(T^{b}\right)=\bigcap_{i} \mathcal{F}_{i}\left(T^{b}\right)$. Note, $\mathcal{F}\left(T^{a}\right) \times \mathcal{F}\left(T^{b}\right)$ is a subsigma algebra on $\mathcal{S}\left(T^{a}\right) \times \mathcal{S}\left(T^{b}\right)$. Indeed, it is closed under $\mathcal{T}$. With this in mind, we call $\mathcal{F}\left(T^{a}\right) \times \mathcal{F}\left(T^{b}\right)$ the coarsest sigma-algebra closed under $\mathcal{T}$.

Definition 5.2 Call a $(\Theta, \mathcal{S}(\Theta))$-based structure $\mathcal{T}=\left\langle(\Theta, \mathcal{S}(\Theta)) ;\left(T^{a}, \mathcal{S}\left(T^{a}\right)\right),\left(T^{b}, \mathcal{S}\left(T^{b}\right)\right) ; \beta^{a}, \beta^{b}\right\rangle$ strongly measurable if $\mathcal{S}\left(T^{a}\right) \times \mathcal{S}\left(T^{b}\right)$ is the coarsest sigma algebra that is closed under $\mathcal{T}$.

Now we can state the main result.

Theorem 5.1 Fix a $(\Theta, \mathcal{S}(\Theta))$-based structure $\mathcal{T}_{*}$.

(i) Suppose $\mathcal{T}_{*}$ is strongly measurable. Than, for each $(\Theta, \mathcal{S}(\Theta))$-based structure $\mathcal{T},\left(h^{a}, h^{b}\right)$ is a hierarchy morphism from $\mathcal{T}$ to $\mathcal{T}_{*}$ if and only if $\left(h^{a}, h^{b}\right)$ is also a type morphism from $\mathcal{T}$ to $\mathcal{T}_{*}$.

(ii) Suppose that, for each $(\Theta, \mathcal{S}(\Theta))$-based structure $\mathcal{T},\left(h^{a}, h^{b}\right)$ is a hierarchy morphism from $\mathcal{T}$ to $\mathcal{T}_{*}$ if and only if $\left(h^{a}, h^{b}\right)$ is also a type morphism from $\mathcal{T}$ to $\mathcal{T}_{*} . \quad$ Then, $\mathcal{T}_{*}$ is strongly measurable.

Theorem 5.1 says that strong measurability is a necessary and sufficient condition for each hierarchy morphism to be a type morphism. The condition of strong measurability is a defined on the type structure alone. So, to check whether a particular structure $\mathcal{T}_{*}$ is strongly measurable, we do not need to leave the domain of hierarchies of beliefs - we simply need to check whether 
$\mathcal{S}\left(T_{*}^{a}\right) \times \mathcal{S}\left(T_{*}^{b}\right)$ is the coarsest sigma algebra that is closed under $\mathcal{T}_{*}$. Referring back to Example 4.2 we note that there are two subsigma algebras that are closed under $\mathcal{T}_{*}$, namely $\mathcal{S}\left(T_{*}^{a}\right) \times \mathcal{S}\left(T_{*}^{b}\right)$ and $\left\{\emptyset, T_{*}^{a}\right\} \times\left\{\emptyset, T_{*}^{b}\right\}$. So, here, $\mathcal{F}\left(T_{*}^{a}\right) \times \mathcal{F}\left(T_{*}^{b}\right)$ is $\left\{\emptyset, T_{*}^{a}\right\} \times\left\{\emptyset, T_{*}^{b}\right\}$, and so the structure $\mathcal{T}_{*}$ is not strongly measurable.

Note, precisely because strong measurability is defined within the domain of type structures, we are left with the question of how to interpret the condition. What are we assuming about the players' language (i.e., the players' sigma-algebras), when we presume a type structure is strongly measurable? Refer back to Example 4.2 There we have that $\mathcal{F}\left(T_{*}^{a}\right) \times \mathcal{F}\left(T_{*}^{b}\right)$ is $\left\{\emptyset, T_{*}^{a}\right\} \times\left\{\emptyset, T_{*}^{b}\right\}$ and this is precisely the sigma algebra generated by the hierarchies of beliefs. Indeed, as our discussion in Section 4 suggested, this holds more generally. Strong measurability is equivalent to the requirement that the hierarchies of beliefs determine the measurable sets of types. So, substantively, strong measurability is the requirement that each player's language in type space corresponds exactly to the player's language in hierarchy space.

Given a $\left(\Theta, \mathcal{S}(\Theta)\right.$ )-based structure $\mathcal{T}$, recall, $\sigma\left(\delta^{a}\right)$ is the sigma algebra on $T^{a}$ generated by the map $\delta^{a}$, i.e., $F^{a} \in \sigma\left(\delta^{a}\right)$ if and only if there is some event $E \in \mathcal{S}\left(\prod_{m=1}^{\infty} \Delta\left(Z_{m}\right)\right)$ with $F^{a}=$ $\left(\delta^{a}\right)^{-1}(E)$. And, likewise, define $\sigma\left(\delta^{b}\right)$.

Proposition 5.1 A $(\Theta, \mathcal{S}(\Theta))$-based structure $\mathcal{T}=\left\langle(\Theta, \mathcal{S}(\Theta)) ;\left(T^{a}, \mathcal{S}\left(T^{a}\right)\right),\left(T^{b}, \mathcal{S}\left(T^{b}\right)\right) ; \beta^{a}, \beta^{b}\right\rangle$ is strongly measurable if and only if $\mathcal{S}\left(T^{a}\right)=\sigma\left(\delta^{a}\right)$ and $\mathcal{S}\left(T^{b}\right)=\sigma\left(\delta^{b}\right)$.

Proposition 5.1 is inspired by Mertens-Zamir [17, page 6; 1985]. To the best of our knowledge, it is new to the literature. In Section 7, we return to discuss sufficient conditions for a type structure to be strongly measurable. First, however, we give the proof of Theorem 5.1 .

\section{Proof of Theorem 5.1}

We begin with the proof of Proposition 5.1. We will then use Proposition 5.1 to prove Theorem 5.1 To show this, it suffices to show that, for any $(\Theta, \mathcal{S}(\Theta))$-based structure $\mathcal{T}, \sigma\left(\delta^{a}\right) \times \sigma\left(\delta^{b}\right)$ is the smallest sigma-algebra closed under $\mathcal{T}$. We proceed to build toward this result.

It will be useful to introduce some further notation. Let $Z=Z_{1} \times \prod_{m=1}^{\infty} \Delta\left(Z_{m}\right)$. Consider a structure $\mathcal{T}$ and define $\rho^{a}: \Theta \times T^{b} \rightarrow Z$ (resp. $\rho^{b}: \Theta \times T^{a} \rightarrow Z$ ) so that $\rho^{a}\left(\theta, t^{b}\right)=\left(\theta, \delta^{b}\left(t^{b}\right)\right)$ (resp. $\left.\rho^{b}\left(\theta, t^{a}\right)=\left(\theta, \delta^{a}\left(t^{a}\right)\right)\right)$. And define $\rho_{*}^{a}, \rho_{*}^{b}$ for the structure $\mathcal{T}_{*}$ analogously. Note, each of these maps is a product of two measurable maps and so measurable.

Note, the mapping $\underline{\rho}^{a}: \Delta\left(\Theta \times T^{b}\right) \rightarrow \Delta(Z)$ is measurable. As such, the mapping $\left(\underline{\rho}^{a} \circ \beta^{a}\right)$ : $T^{a} \rightarrow \Delta(Z)$ is also measurable. We begin by relating the map $\left(\underline{\rho}^{a} \circ \beta^{a}\right)$ to each of the maps $\delta_{m}^{a}=\underline{\rho}_{m}^{a} \circ \beta^{a}$. In the course of doing so, we use the fact that $Z_{m+1}=Z_{1} \times \prod_{n=1}^{m} \Delta\left(Z_{n}\right)$.

Lemma 6.1 Fix a structure $\mathcal{T}$. For any $m$, the following hold.

(i) For each $H_{m} \in \mathcal{S}\left(Z_{m}\right),\left(\rho_{m}^{a}\right)^{-1}\left(H_{m}\right)=\left(\rho^{a}\right)^{-1}\left(H_{m} \times \prod_{n=m}^{\infty} \Delta\left(Z_{n}\right)\right)$. 
(ii) For each $t^{a} \in T^{a}, \delta_{m}^{a}\left(t^{a}\right)=\operatorname{marg}_{Z_{m}} \underline{\rho}^{a}\left(\beta^{a}\left(t^{a}\right)\right)$.

And, likewise, with a and $b$ reversed.

Proof. Part (i) is immediate from the definitions. For Part (ii), fix an event $H_{m}$ in $Z_{m}=$ $Z_{1} \times \prod_{n=1}^{m-1} \Delta\left(Z_{n}\right)$, and note that

$$
\begin{aligned}
\delta_{m}^{a}\left(t^{a}\right)\left(H_{m}\right) & =\beta^{a}\left(t^{a}\right)\left(\left(\rho_{m}^{a}\right)^{-1}\left(H_{m}\right)\right) \\
& =\beta^{a}\left(t^{a}\right)\left(\left(\rho^{a}\right)^{-1}\left(H_{m} \times \prod_{n=m}^{\infty} \Delta\left(Z_{n}\right)\right)\right) \\
& =\operatorname{marg}_{Z_{m}} \underline{\rho}^{a}\left(\beta^{a}\left(t^{a}\right)\right)\left(H_{m}\right),
\end{aligned}
$$

where the second line uses Part (i)

Given measurable spaces $\left(\Omega_{1}, \mathcal{S}\left(\Omega_{1}\right)\right)$ and $\left(\Omega_{2}, \mathcal{S}\left(\Omega_{2}\right)\right)$, write $\mathcal{S}\left(\Omega_{1}\right) \times \mathcal{S}\left(\Omega_{2}\right)$ for the product sigma-algebra (of $\mathcal{S}\left(\Omega_{1}\right)$ and $\mathcal{S}\left(\Omega_{2}\right)$ ) on $\Omega_{1} \times \Omega_{2}$.

Remark 6.1 Fix measurable spaces $\left(\Omega_{1}, \mathcal{S}\left(\Omega_{1}\right)\right),\left(\Omega_{2}, \mathcal{S}\left(\Omega_{2}\right)\right),\left(\Phi_{1}, \mathcal{S}\left(\Phi_{2}\right)\right)$, and $\left(\Phi_{2}, \mathcal{S}\left(\Phi_{2}\right)\right)$. Fix also measurable maps $f_{1}: \Omega_{1} \rightarrow \Phi_{1}$ and $f_{2}: \Omega_{2} \rightarrow \Phi_{2}$, with $\sigma\left(f_{1}\right)=\mathcal{S}\left(\Omega_{1}\right)$ and $\sigma\left(f_{2}\right)=\mathcal{S}\left(\Omega_{2}\right)$. Then, $\sigma\left(f_{1} \times f_{2}\right)=\mathcal{S}\left(\Omega_{1}\right) \times \mathcal{S}\left(\Omega_{2}\right)$.

Lemma 6.2 For any $(\Theta, \mathcal{S}(\Theta))$-based structure $\mathcal{T}, \sigma\left(\delta^{a}\right) \times \sigma\left(\delta^{b}\right)$ is closed with respect to $\mathcal{T}$.

Proof. We make use of two facts. First, by Remark 6.1. $\sigma\left(\rho^{a}\right)=\mathcal{S}(\Theta) \times \sigma\left(\delta^{b}\right)$. Second, $\mathcal{S}(Z)$ is generated by the algebra that consists of sets of the form $H_{m} \times \prod_{n=m}^{\infty} \Delta\left(Z_{n}\right)$, for $m \geq 1$ and $H_{m} \in \mathcal{S}\left(Z_{m}\right)$. So, using Lemma 2.1 in Heifetz-Samet [13, 1999], it suffices to show that, for each $H_{m} \in \mathcal{S}\left(Z_{m}\right)$ and each $p \in[0,1]$,

$$
\left(\beta^{a}\right)^{-1}\left(\left\{\mu \in \Delta\left(\Theta \times T^{b}\right): \mu\left(\left(\rho^{a}\right)^{-1}\left(H_{m} \times \prod_{n=m}^{\infty} \Delta\left(Z_{n}\right)\right)\right) \geq p\right\}\right)
$$

is contained in $\sigma\left(\delta^{a}\right)$.

Fix some $H_{m} \in \mathcal{S}\left(Z_{m}\right)$ and some $p \in[0,1]$. We will show that

$$
\left(\beta^{a}\right)^{-1}\left(\left\{\mu \in \Delta\left(\Theta \times T^{b}\right): \mu\left(\left(\rho^{a}\right)^{-1}\left(H_{m} \times \prod_{n=m}^{\infty} \Delta\left(Z_{n}\right)\right)\right) \geq p\right\}\right)=\left\{t^{a} \in T^{a}: \delta_{m}^{a}\left(t^{a}\right)\left(H_{m}\right) \geq p\right\} .
$$

If Equation 1 holds, we are done: By definition, $\delta^{a}: T^{a} \rightarrow \prod_{m=1}^{\infty} \Delta\left(Z_{m}\right)$, is $\sigma\left(\delta^{a}\right)$-measurable. Since $\prod_{m=1}^{\infty} \Delta\left(Z_{m}\right)$ is endowed with the product sigma-algebra, $\delta_{m}^{a}: T^{a} \rightarrow \Delta\left(Z_{m}\right)$ is also $\sigma\left(\delta^{a}\right)$ measurable. It follows that

$$
\left\{t^{a} \in T^{a}: \delta_{m}^{a}\left(t^{a}\right)\left(H_{m}\right) \geq p\right\}=\left(\delta_{m}^{a}\right)^{-1}\left(\left\{\nu_{m} \in \Delta\left(Z_{m}\right): \nu_{m}\left(H_{m}\right) \geq p\right\}\right)
$$

is contained in $\sigma\left(\delta^{a}\right)$. So, we get that the left-hand side of Equation 1 is also contained in $\sigma\left(\delta^{a}\right)$. 
Now we turn to show Equation 1. Fix a type $t^{a} \in T^{a}$ and note that

$$
\begin{aligned}
\delta_{m}^{a}\left(t^{a}\right)\left(H_{m}\right) & =\operatorname{marg}_{Z_{m}}\left(\underline{\rho}^{a}\left(\beta^{a}\left(t^{a}\right)\right)\right)\left(H_{m}\right) \\
& =\left(\underline{\rho}^{a}\left(\beta^{a}\left(t^{a}\right)\right)\right)\left(H_{m} \times \prod_{n=m}^{\infty} \Delta\left(Z_{n}\right)\right) \\
& =\left(\beta^{a}\left(t^{a}\right)\right)\left(\left(\rho^{a}\right)^{-1}\left(H_{m} \times \prod_{n=m}^{\infty} \Delta\left(Z_{n}\right)\right)\right)
\end{aligned}
$$

where the second line uses Lemma 6.1(ii), It follows that

$$
\begin{aligned}
\left\{t^{a} \in T^{a}: \delta_{m}^{a}\left(t^{a}\right)\left(H_{m}\right) \geq p\right\} & =\left\{t^{a} \in T^{a}:\left(\beta^{a}\left(t^{a}\right)\right)\left(\left(\rho^{a}\right)^{-1}\left(H_{m} \times \prod_{n=m}^{\infty} \Delta\left(Z_{n}\right)\right)\right) \geq p\right\} \\
& =\left(\beta^{a}\right)^{-1}\left(\left\{\mu \in \Delta\left(\Theta \times T^{b}\right): \mu\left(\left(\rho^{a}\right)^{-1}\left(H_{m} \times \prod_{n=m}^{\infty} \Delta\left(Z_{n}\right)\right)\right) \geq p\right\}\right)
\end{aligned}
$$

as required.

Lemma 6.3 For any $(\Theta, \mathcal{S}(\Theta))$-based structure $\mathcal{T}=\left\langle(\Theta, \mathcal{S}(\Theta)) ;\left(T^{a}, \mathcal{S}\left(T^{a}\right)\right),\left(T^{b}, \mathcal{S}\left(T^{b}\right)\right) ; \beta^{a}, \beta^{b}\right\rangle$, $\sigma\left(\delta^{a}\right) \times \sigma\left(\delta^{b}\right) \subseteq \mathcal{F}\left(T^{a}\right) \times \mathcal{F}\left(T^{a}\right)$.

Proof. Fix a $(\Theta, \mathcal{S}(\Theta))$-based structure

$$
\mathcal{T}=\left\langle(\Theta, \mathcal{S}(\Theta)) ;\left(T^{a}, \mathcal{S}\left(T^{a}\right)\right),\left(T^{b}, \mathcal{S}\left(T^{b}\right)\right) ; \beta^{a}, \beta^{b}\right\rangle .
$$

We will construct a new $(\Theta, \mathcal{S}(\Theta))$-based structure

$$
\mathcal{T}_{*}=\left\langle(\Theta, \mathcal{S}(\Theta)) ;\left(T^{a}, \mathcal{F}\left(T^{a}\right)\right),\left(T^{b}, \mathcal{F}\left(T^{b}\right)\right) ; \beta_{*}^{a}, \beta_{*}^{b}\right\rangle,
$$

so that, for each $t^{a} \in T^{a}$ and each $E^{b} \in \mathcal{S}(\Theta) \times \mathcal{F}\left(T^{b}\right), \beta_{*}^{a}\left(t^{a}\right)\left(E^{b}\right)=\beta^{a}\left(t^{a}\right)\left(E^{b}\right)$. (This is welled defined, since $\mathcal{S}(\Theta) \times \mathcal{F}\left(T^{b}\right) \subseteq \mathcal{S}(\Theta) \times \mathcal{S}\left(T^{b}\right)$.) And likewise with $a$ and $b$ interchanged. Note, since $\mathcal{F}\left(T^{a}\right) \times \mathcal{F}\left(T^{b}\right)$ is closed under $\mathcal{T}$, it follows that $\beta_{*}^{a}$ and $\beta_{*}^{b}$ are measurable. So, $\mathcal{I}_{*}$ is indeed a well-defined type structure.

It suffices to show that $\delta_{*}^{a}=\delta^{a}$ and $\delta_{*}^{b}=\delta^{b}$. If so, then $\sigma\left(\delta^{a}\right) \times \sigma\left(\delta^{b}\right)=\sigma\left(\delta_{*}^{a}\right) \times \sigma\left(\delta_{*}^{b}\right)$. Then, using the fact that $\delta_{*}^{a}$ and $\delta_{*}^{b}$ are measurable, we have that

$$
\sigma\left(\delta^{a}\right) \times \sigma\left(\delta^{b}\right)=\sigma\left(\delta_{*}^{a}\right) \times \sigma\left(\delta_{*}^{b}\right) \subseteq \mathcal{F}\left(T^{a}\right) \times \mathcal{F}\left(T^{b}\right)
$$

as required.

Now, we show that $\delta_{*}^{a}=\delta^{a}$ and $\delta_{*}^{b}=\delta^{b}$. Specifically, we show that, for each $m$, (i) $\rho_{m, *}^{a}=\rho_{m}^{a}$ and (ii) $\delta_{m, *}^{a}=\delta_{m}^{a}$. And likewise for $b$. Part (i) is immediate for $m=1$. Likewise, part (ii) holds since, for each event $E_{1}$ in $Z_{1}$,

$$
\delta_{1, *}^{a}\left(t^{a}\right)\left(E_{1}\right)=\beta_{*}^{a}\left(t^{a}\right)\left(E_{1} \times T^{b}\right)=\beta^{a}\left(t^{a}\right)\left(E_{1} \times T^{b}\right)=\delta_{1}^{a}\left(t^{a}\right)\left(E_{1}\right) .
$$

Now assume parts (i) and (ii) hold for $m$. Then, using the induction hypothesis, part (i) holds for 
$m+1$. Fix an event $E_{m+1}$ in $Z_{m+1}$. Then

$$
\begin{aligned}
\delta_{m+1, *}^{a}\left(t^{a}\right)\left(E_{m+1}\right) & =\beta_{*}^{a}\left(t^{a}\right)\left(\left(\rho_{m+1, *}^{a}\right)^{-1}\left(E_{m+1}\right)\right) \\
& =\beta^{a}\left(t^{a}\right)\left(\left(\rho_{m+1}^{a}\right)^{-1}\left(E_{m+1}\right)\right)=\delta_{m+1}^{a}\left(t^{a}\right)\left(E_{m+1}\right),
\end{aligned}
$$

as required.

Lemma 6.4 For any $(\Theta, \mathcal{S}(\Theta))$-based structure $\mathcal{T}=\left\langle(\Theta, \mathcal{S}(\Theta)) ;\left(T^{a}, \mathcal{S}\left(T^{a}\right)\right),\left(T^{b}, \mathcal{S}\left(T^{b}\right)\right) ; \beta^{a}, \beta^{b}\right\rangle$, $\sigma\left(\delta^{a}\right) \times \sigma\left(\delta^{b}\right)=\mathcal{F}\left(T^{a}\right) \times \mathcal{F}\left(T^{a}\right)$.

Proof. By Lemma 6.2, $\mathcal{F}\left(T^{a}\right) \times \mathcal{F}\left(T^{a}\right) \subseteq \sigma\left(\delta^{a}\right) \times \sigma\left(\delta^{b}\right)$. By Lemma 6.3, $\sigma\left(\delta^{a}\right) \times \sigma\left(\delta^{b}\right) \subseteq$ $\mathcal{F}\left(T^{a}\right) \times \mathcal{F}\left(T^{a}\right)$

Proof of Proposition 5.1. Immediate from Lemma 6.4.

We now turn to the proof of Theorem 5.1(i). In light of Proposition 5.1 and Proposition 3.1 it suffices to show the following.

Lemma 6.5 Fix $(\Theta, \mathcal{S}(\Theta))$-based structure $\mathcal{T}_{*}=\left\langle(\Theta, \mathcal{S}(\Theta)) ;\left(T_{*}^{a}, \sigma\left(\delta_{*}^{a}\right)\right),\left(T_{*}^{b}, \sigma\left(\delta_{*}^{b}\right)\right) ; \beta_{*}^{a}, \beta_{*}^{b}\right\rangle$. For each $(\Theta, \mathcal{S}(\Theta))$-based structure $\mathcal{T},\left(h^{a}, h^{b}\right)$ is a hierarchy morphism from $\mathcal{T}$ to $\mathcal{T}_{*}$ only if $\left(h^{a}, h^{b}\right)$ is also a type morphism from $\mathcal{T}$ to $\mathcal{T}_{*}$.

To show Lemma 6.5, we begin by showing that if $T_{*}^{a}$ and $T_{*}^{b}$ are endowed with the sigma algebra generated by hierarchies, then each hierarchy morphism is measurable map. A key step is relating the events in $T_{*}^{a}$ generated by $h^{a}$ to events in $T_{*}^{a}$ generated by $\delta_{*}^{a}$. The following Lemma will be of use for this step.

Lemma 6.6 Fix two $(\Theta, \mathcal{S}(\Theta))$-based structures $\mathcal{T}$ and $\mathcal{T}_{*}$, so that there is a hierarchy morphism, viz. $\left(h^{a}, h^{b}\right)$, from $\mathcal{T}$ to $\mathcal{T}_{*}$. If $E_{*} \in \mathcal{S}\left(T_{*}^{a}\right)$ and $H \in \mathcal{S}\left(\prod_{m=1}^{\infty} \Delta\left(Z_{m}\right)\right)$ are events satisfying $\left(\delta_{*}^{a}\right)^{-1}(H)=E_{*}$, then $\left(h^{a}\right)^{-1}\left(E_{*}\right)=\left(\delta^{a}\right)^{-1}(H)$.

Proof. Note, $t^{a} \in\left(h^{a}\right)^{-1}\left(E_{*}\right)$ if and only if $h^{a}\left(t^{a}\right) \in E_{*}$. By assumption, $h^{a}\left(t^{a}\right) \in E_{*}$ if and only if $\delta_{*}^{a}\left(h^{a}\left(t^{a}\right)\right) \in H$. Since $h^{a}$ is a hierarchy morphism, $\delta_{*}^{a}\left(h^{a}\left(t^{a}\right)\right) \in H$ if and only if $\delta^{a}\left(t^{a}\right) \in H$. Finally, $\delta^{a}\left(t^{a}\right) \in H$ if and only if $t^{a} \in\left(\delta^{a}\right)^{-1}(H)$. Putting this together, $\left(h^{a}\right)^{-1}\left(E_{*}\right)=\left(\delta^{a}\right)^{-1}(H)$, as desired.

Lemma 6.7 Fix two $(\Theta, \mathcal{S}(\Theta))$-based structures $\mathcal{T}$ and $\mathcal{T}_{*}$, so that there is a hierarchy morphism, viz. $\left(h^{a}, h^{b}\right)$, from $\mathcal{T}$ to $\mathcal{T}_{*}$. If $\left(T_{*}^{a}, \mathcal{S}\left(T_{*}^{a}\right)\right)=\left(T_{*}^{a}, \sigma\left(\delta_{*}^{a}\right)\right)$ and $\left(T_{*}^{b}, \mathcal{S}\left(T_{*}^{b}\right)\right)=\left(T_{*}^{b}, \sigma\left(\delta_{*}^{b}\right)\right)$, then $h^{a}$ and $h^{b}$ are measurable.

Proof. Fix an event $E_{*}$ in $T_{*}^{a}$. We will show that $\left(h^{a}\right)^{-1}\left(E_{*}\right) \in \mathcal{S}\left(T^{a}\right)$.

Since $\left(T_{*}^{a}, \mathcal{S}\left(T_{*}^{a}\right)\right)=\left(T_{*}^{a}, \sigma\left(\delta_{*}^{a}\right)\right)$, there is some event $H \in \mathcal{S}\left(\prod_{m=1}^{\infty} \Delta\left(Z_{m}\right)\right)$ with $\left(\delta_{*}^{a}\right)^{-1}(H)=$ $E_{*}$. By Lemma 6.6. $\left(h^{a}\right)^{-1}\left(E_{*}\right)=\left(\delta^{a}\right)^{-1}(H)$. Now, the result follows from the fact that $\delta^{a}$ is measurable. 
(The measurability of $h^{b}$ is similarly established.)

We now proceed to show that a hierarchy morphism, viz. $\left(h^{a}, h^{b}\right)$, satisfies the second condition of a type morphism-namely, that $\underline{\mathrm{id} \times h^{b}} \circ \beta^{a}=\beta_{*}^{a} \circ h^{a}$ and $\underline{\mathrm{id} \times h^{a}} \circ \beta^{b}=\beta_{*}^{b} \circ h^{b}$.

Lemma 6.8 Suppose $\left(h^{a}, h^{b}\right)$ is a hierarchy morphism from $\mathcal{T}$ to $\mathcal{T}_{*}$. Then, for any $t^{a} \in T^{a}$, $\underline{\rho}^{a}\left(\beta^{a}\left(t^{a}\right)\right)=\underline{\rho}_{*}^{a}\left(\beta_{*}^{a}\left(h^{a}\left(t^{a}\right)\right)\right)$.

Proof. Fix $t^{a} \in T^{a}$. By definition of a hierarchy morphism,

$$
\left(\delta_{1}^{a}\left(t^{a}\right), \delta_{2}^{a}\left(t^{a}\right), \ldots\right)=\left(\delta_{1, *}^{a}\left(h^{a}\left(t^{a}\right)\right), \delta_{2, *}^{a}\left(h^{a}\left(t^{a}\right)\right), \ldots\right)
$$

So, using Lemma 6.1 ,

$\left(\operatorname{marg}_{Z_{1}} \underline{\rho}^{a}\left(\beta^{a}\left(t^{a}\right)\right), \operatorname{marg}_{Z_{2}} \underline{\rho}^{a}\left(\beta^{a}\left(t^{a}\right)\right), \ldots\right)=\left(\operatorname{marg}_{Z_{1}} \underline{\rho}_{*}^{a}\left(\beta^{a}\left(h^{a}\left(t^{a}\right)\right)\right), \operatorname{marg}_{Z_{2}} \underline{\rho}_{*}^{a}\left(\beta^{a}\left(h^{a}\left(t^{a}\right)\right)\right), \ldots\right)$.

That is, $\underline{\rho}^{a}\left(\beta^{a}\left(t^{a}\right)\right)$ and $\underline{\rho}_{*}^{a}\left(\beta_{*}^{a}\left(h^{a}\left(t^{a}\right)\right)\right)$ agree on all sets of the form $H_{m} \times \prod_{n=m}^{\infty} \Delta\left(Z_{n}\right) \subseteq Z_{m} \times$ $\prod_{n=m}^{\infty} \Delta\left(Z_{n}\right)$. Since these sets form a semialgebra that generates $\mathcal{S}(Z)$, Theorem 21.10d in Yeh [23, 2000] gives that $\underline{\rho}^{a}\left(\beta^{a}\left(t^{a}\right)\right)=\underline{\rho}_{*}^{a}\left(\beta_{*}^{a}\left(h^{a}\left(t^{a}\right)\right)\right)$.

Lemma 6.9 Fix two $(\Theta, \mathcal{S}(\Theta))$-based structures $\mathcal{T}$ and $\mathcal{T}_{*}$, so that there is a hierarchy morphism, viz. $\left(h^{a}, h^{b}\right)$, from $\mathcal{T}$ to $\mathcal{T}_{*}$. If $E_{*} \in \mathcal{S}\left(\Theta \times T_{*}^{a}\right)$ and $H \in \mathcal{S}(Z)$ are events satisfying $\left(\rho_{*}^{b}\right)^{-1}(H)=$ $E_{*}$, then $\left(\mathrm{id} \times h^{a}\right)^{-1}\left(E_{*}\right)=\left(\rho^{b}\right)^{-1}(H)$.

Proof. Note, $\left(\theta, t^{a}\right) \in\left(\text { id } \times h^{a}\right)^{-1}\left(E_{*}\right)$ if and only if $\left(\theta, h^{a}\left(t^{a}\right)\right) \in E_{*}$. By assumption, $\left(\theta, h^{a}\left(t^{a}\right)\right) \in$ $E_{*}$ if and only if $\rho_{*}^{b}\left(\theta, h^{a}\left(t^{a}\right)\right) \in H$, which holds if and only if $\left(\theta, \delta_{*}^{a}\left(h^{a}\left(t^{a}\right)\right) \in H\right.$. Now, since $h^{a}$ is a hierarchy morphism, $\left(\theta, \delta_{*}^{a}\left(h^{a}\left(t^{a}\right)\right) \in H\right.$ if and only if $\left(\theta, \delta^{a}\left(t^{a}\right)\right) \in H$. Using the definition of $\rho^{b},\left(\theta, \delta^{a}\left(t^{a}\right)\right) \in H$ if and only if $\rho^{b}\left(\theta, t^{a}\right) \in H$, i.e., if and only if $\left(\theta, t^{a}\right) \in\left(\rho^{b}\right)^{-1}(H)$. Hence, $\left(\mathrm{id} \times h^{a}\right)^{-1}\left(E_{*}\right)=\left(\rho^{b}\right)^{-1}(H)$.

\section{Proof of Lemma 6.5.}

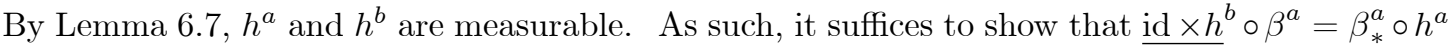

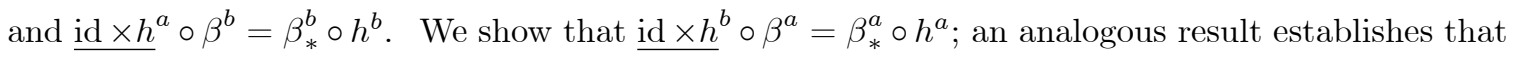

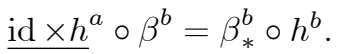

Fix an event $E_{*}$ in $\Theta \times T_{*}^{b}$. We need to show that

$$
\beta^{a}\left(t^{a}\right)\left(\left(\mathrm{id} \times h^{b}\right)^{-1}\left(E_{*}\right)\right)=\beta_{*}^{a}\left(h^{a}\left(t^{a}\right)\right)\left(E_{*}\right) .
$$

To do so, first note that we can find an event $H \in \mathcal{S}(Z)$ so that $\left(\rho_{*}^{a}\right)^{-1}(H)=E_{*}$. (This follows from the fact that $\left(T_{*}^{b}, \mathcal{S}\left(T_{*}^{b}\right)\right)=\left(T_{*}^{b}, \sigma\left(\delta_{*}^{b}\right)\right)$ and that we endow the product of measurable sets with the product sigma-algebra.) By Lemma 6.9, we also have that $\left(\rho^{a}\right)^{-1}(H)=\left(\operatorname{id} \times h^{b}\right)^{-1}\left(E_{*}\right)$. 
Thus,

$$
\begin{aligned}
\beta^{a}\left(t^{a}\right)\left(\left(\mathrm{id} \times h^{b}\right)^{-1}\left(E_{*}\right)\right) & =\beta^{a}\left(t^{a}\right)\left(\left(\rho^{a}\right)^{-1}(H)\right) \\
& =\beta_{*}^{a}\left(h^{a}\left(t^{a}\right)\right)\left(\left(\rho_{*}^{a}\right)^{-1}(H)\right) \\
& =\beta_{*}^{a}\left(h^{a}\left(t^{a}\right)\right)\left(E_{*}\right),
\end{aligned}
$$

where the second line follows from Lemma 6.8. This establishes the result.

Now we turn to necessity.

Lemma 6.10 Fix $(\Theta, \mathcal{S}(\Theta))$-based structure $\mathcal{T}_{*}$ so that, for each $(\Theta, \mathcal{S}(\Theta))$-based structure $\mathcal{T}$, $\left(h^{a}, h^{b}\right)$ is a hierarchy morphism from $\mathcal{T}$ to $\mathcal{T}_{*}$ only if $\left(h^{a}, h^{b}\right)$ is also a type morphism from $\mathcal{T}$ to $\mathcal{T}_{*}$. Then $\left(T_{*}^{a}, \mathcal{S}\left(T_{*}^{a}\right)\right)=\left(T_{*}^{a}, \sigma\left(\delta_{*}^{a}\right)\right)$ and $\left(T_{*}^{b}, \mathcal{S}\left(T_{*}^{b}\right)\right)=\left(T_{*}^{b}, \sigma\left(\delta_{*}^{b}\right)\right)$.

Proof. Fix some $\mathcal{T}_{*}$ and suppose, contra hypothesis, that $\mathcal{S}\left(T_{*}^{a}\right) \neq \sigma\left(\delta_{*}^{a}\right)$. Construct the structure $\mathcal{T}$ as follows. Choose $\left(T^{a}, \mathcal{S}\left(T^{a}\right)\right)=\left(T_{*}^{a}, \sigma\left(\delta_{*}^{a}\right)\right)$ and $\left(T^{b}, \mathcal{S}\left(T^{b}\right)\right)=\left(T_{*}^{b}, \sigma\left(\delta_{*}^{b}\right)\right)$. For each $t^{a} \in T^{a}$, define $\beta^{a}\left(t^{a}\right)$ as the restriction of $\beta_{*}^{a}\left(t^{a}\right)$ to the product sigma-algebra $\mathcal{S}(\Theta) \times \sigma\left(\delta_{*}^{b}\right)$. Define $\beta^{b}$ analogously. It follows from Lemma 6.2 that $\beta^{a}$ and $\beta^{b}$ are measurable. As such, $\mathcal{T}$ is indeed a type structure.

Consider the identity maps $h^{a}: T_{*}^{a} \rightarrow T_{*}^{a}$ and $h^{b}: T_{*}^{b} \rightarrow T_{*}^{b}$. Then $\left(h^{a}, h^{b}\right)$ is a hierarchy morphism from $\mathcal{T}$ to $\mathcal{T}_{*}$. But, there is some $E_{*} \in \mathcal{S}\left(T_{*}^{a}\right) \backslash \sigma\left(\delta_{*}^{a}\right)$. So, $\left(h^{a}\right)^{-1}\left(E_{*}\right) \notin \sigma\left(\delta_{*}^{a}\right)$ and so $h^{a}$ is not measurable.

Proof of Theorem 5.1. Part (i) follows from Proposition 5.1. Lemma 6.5, and Proposition 3.1. Part (ii) follows from Proposition 5.1, Lemma 6.10, and Proposition 3.1.

\section{$7 \quad$ When Are Type Structures Strongly Measurable?}

Theorem 5.1 says there is an equivalence between hierarchy and type morphisms if and only if the structure is strongly measurable. This raises the question: Are there interesting cases, where the type structures are necessarily strongly measurable?

We will provide sufficient conditions that guarantee strong measurability. To gain intuition for these conditions, refer to Example 4.1. One possibility is to consider the case where the measurable structures of hierarchies and types (in $\mathcal{T}_{*}$ ) are both generated by the same underlying topology, e.g., they are both Polish or both compact metrizable, etc.... Example 4.2 tells us that this will not be enough. There, the measurable structures of hierarchies and types (in $\mathcal{T}_{*}$ ) are both generated by a compact metrizable structure. But, as already seen, $\mathcal{T}_{*}$ is not strongly measurable. The reason is that singletons are contained in $\mathcal{S}\left(T_{*}^{b}\right)$, despite the fact that they induce the same hierarchies of beliefs. Put differently, the reason is that, in this case, two types induce the same hierarchies of beliefs, but are in different measurable sets. 
We will provide conditions on a type structure so that we do get a strongly measurable structure. These conditions will be both related to the underlying measurable structure and redundancies in the type structure (i.e., types that induce the same hierarchies of beliefs). Building on results in Mertens-Zamir [17, Proposition 2.5; 1985] and Purves [19, 1966], these conditions can be stated in terms of the type structure $\mathcal{T}_{*}$ alone.

Let us begin with the idea of redundancy.

Definition 7.1 (Mertens-Zamir [17, page 6; 1985]) Call a $(\Theta, \mathcal{S}(\Theta))$-based structure $\mathcal{T}$ nonredundant if the associated maps $\delta^{a}$ and $\delta^{b}$ are injective.

Definition 7.2 Call a $(\Theta, \mathcal{S}(\Theta))$-based structure $\mathcal{T}$ measurably non-redundant if the associated maps $\delta^{a}$ and $\delta^{b}$ satisfy the following condition: For each $t^{a} \in T^{a}$ and each $E \in \mathcal{S}\left(T^{a}\right)$, either $\left(\delta^{a}\right)^{-1}\left(\left\{\delta^{a}\left(t^{a}\right)\right\}\right) \subseteq E$ or $\left(\delta^{a}\right)^{-1}\left(\left\{\delta^{a}\left(t^{a}\right)\right\}\right) \cap E=\emptyset$, and likewise for $b$.

A structure is non-redundant if no two types induce the same hierarchies of beliefs. A structure is measurably non-redundant if, whenever two types induce the same hierarchies of beliefs, they cannot be separated by measurable sets. Of course, a non-redundant structure is measurably non-redundant, but the converse need not hold. In the case where each type is measurable (i.e., $\mathcal{S}\left(T^{a}\right)$ and $\mathcal{S}\left(T^{b}\right)$ contain all the singletons), $\mathcal{T}$ is measurably non-redundant if and only if it is non-redundant.

We will consider one further condition on the structure. First, a mathematical definition. Fix $(\Omega, \mathcal{S}(\Omega)),(\Phi, \mathcal{S}(\Phi))$, and a map $f: \Omega \rightarrow \Phi$. Call $f$ bimeasurable if $f$ is measurable and, for any event $E \in \mathcal{S}(\Omega), f(E) \in \mathcal{S}(\Phi)$.

Definition 7.3 (Brandenburger-Friedenberg [6, Definition 8.2; 2008]) Call a $(\Theta, \mathcal{S}(\Theta))$-based structure $\mathcal{T}$ bimeasurable if the associated maps $\delta^{a}$ and $\delta^{b}$ are bimeasurable.

As it turns out, the bimeasurability condition can also be viewed as a restriction on redundanciesat least in certain cases. We'll come back and talk about this below. But, first, let us point out the connection to strongly measurable structures.

Lemma 7.1 $A(\Theta, \mathcal{S}(\Theta))$-based structure $\mathcal{T}=\left\langle(\Theta, \mathcal{S}(\Theta)) ;\left(T^{a}, \mathcal{S}\left(T^{a}\right)\right),\left(T^{b}, \mathcal{S}\left(T^{b}\right)\right) ; \beta^{a}, \beta^{b}\right\rangle$.

(i) If $\mathcal{T}$ is strongly measurable, then $\mathcal{T}$ is measurably non-redundant.

(ii) If $\mathcal{T}$ is measurably non-redundant and $\mathcal{T}$ is bimeasurable, then $\mathcal{T}$ is strongly measurable.

Proof. Suppose $\mathcal{T}$ is strongly measurable. Fix a type $t^{a} \in T^{a}$ and an event $E \in \mathcal{S}\left(T^{a}\right)$. Since $\mathcal{T}$ is strongly measurable, Proposition 5.1 gives that there exists $F \in \mathcal{S}\left(\prod_{m=1}^{\infty} \Delta\left(Z_{m}\right)\right)$ with $\left(\delta^{a}\right)^{-1}(F)=$ $E$. If $\delta^{a}\left(t^{a}\right) \in F$, then $\left(\delta^{a}\right)^{-1}\left(\left\{\delta^{a}\left(t^{a}\right)\right\}\right) \subseteq\left(\delta^{a}\right)^{-1}(F)=E$. If $\delta^{a}\left(t^{a}\right) \notin F$, then $\left(\delta^{a}\right)^{-1}\left(\left\{\delta^{a}\left(t^{a}\right)\right\}\right) \cap$ $\left(\delta^{a}\right)^{-1}(F)=\emptyset$. So, if $\delta^{a}\left(t^{a}\right) \notin F$, then $\left(\delta^{a}\right)^{-1}\left(\left\{\delta^{a}\left(t^{a}\right)\right\}\right) \cap E=\emptyset$. Applying the argument to $b$ establishes that $\mathcal{T}$ is measurably non-redundant. 
Suppose $\mathcal{T}$ is measurably non-redundant and $\mathcal{T}$ is bimeasurable. We will show $\mathcal{S}\left(T^{a}\right)=\sigma\left(\delta^{a}\right)$. Note, since $\delta^{a}$ is measurable, $\sigma^{a}\left(\delta^{a}\right) \subseteq \mathcal{S}\left(T^{a}\right)$. Fix an event $E \in \mathcal{S}\left(T^{a}\right)$. Since $\delta^{a}$ is bimeasurable, $\delta^{a}(E) \in \mathcal{S}\left(\prod_{m=1}^{\infty} \Delta\left(Z_{m}\right)\right)$. Now note that

$$
\left(\delta^{a}\right)^{-1}\left(\delta^{a}(E)\right) \subseteq E \subseteq\left(\delta^{a}\right)^{-1}\left(\delta^{a}(E)\right)
$$

where the first inclusion follows from measurably non-redundant and the second is standard. Thus, $E=\left(\delta^{a}\right)^{-1}\left(\delta^{a}(E)\right)$ and so contained in $\sigma\left(\delta^{a}\right)$. Applying Proposition 5.1 completes the proof.

Lemma 7.1 states that measurable non-redundancy and bimeasurability are sufficient to guarantee strong measurability. Both of these conditions are defined within the realm of hierarchies of beliefs. The question is whether there are interesting conditions defined on the type structure alone, which guarantee the structure is measurably non-redundant and bimeasurable. We will see that the answer is yes.

Let us begin with the measurably non-redundant condition. As mentioned above, if a type structure is non-redundant, it is measurably non-redundant. And, moreover, when singletons are measurable, the two concepts are equivalent. So, it will be of interest to characterize nonredundancy, as a condition defined on the type structure alone. We can do so, by building on an idea found in Mertens-Zamir [17, Proposition 2.5; 1985].

Fix a measurable space $\left(\Omega, \mathcal{S}(\Omega)\right.$ ). Recall, $\mathcal{S}(\Omega)$ separates points if, for each $\omega, \omega^{\prime} \in \Omega$, there exists some $E \in \mathcal{S}(\Omega)$ with $\omega \in E$ and $\omega^{\prime} \notin E$. The next lemma generalizes Proposition 2.5 in Mertens-Zamir [17, 1985].

Lemma 7.2A $(\Theta, \mathcal{S}(\Theta))$-based structure $\mathcal{T}=\left\langle(\Theta, \mathcal{S}(\Theta)) ;\left(T^{a}, \mathcal{S}\left(T^{a}\right)\right),\left(T^{b}, \mathcal{S}\left(T^{b}\right)\right) ; \beta^{a}, \beta^{b}\right\rangle$ is non-redundant if and only if $\mathcal{F}\left(T^{a}\right)$ and $\mathcal{F}\left(T^{b}\right)$ separate points.

Proof. By Proposition 2 in Liu [15, 2009], $\mathcal{T}$ is non-redundant if and only if $\sigma\left(\delta^{a}\right)$ and $\sigma\left(\delta^{b}\right)$ separate points. So, the result follows from Lemma 6.4 .

Now, let's turn to bimeasurability. For this it will be useful to introduce a definition: Given a topological space $(\Omega, \tau)$, write $\mathcal{B}(\Omega)$ for the set of Borel sets on $\Omega$. Two definitions will be of particular interest. Call $(\Omega, \mathcal{S}(\Omega))$ standard Borel if it is isomorphic to a Polish space-i.e., $(\Omega, \mathcal{S}(\Omega))$ is standard Borel if there is a Polish space $(\Phi, \mathcal{B}(\Phi))$ and a bijective bimeasurable map $f: \Omega \rightarrow \Phi{ }^{6}$ Of course, a Polish space is standard Borel.

Definition 7.4 Call a $(\Theta, \mathcal{S}(\Theta))$-based structure $\mathcal{T}=\left\langle(\Theta, \mathcal{S}(\Theta)) ;\left(T^{a}, \mathcal{S}\left(T^{a}\right)\right),\left(T^{b}, \mathcal{S}\left(T^{b}\right)\right) ; \beta^{a}, \beta^{b}\right\rangle$ standard Borel if $\left(T^{a}, \mathcal{S}\left(T^{a}\right)\right)$, and $\left(T^{b}, \mathcal{S}\left(T^{b}\right)\right)$ are standard Borel.

In the case of a standard Borel structure, the bimeasurability condition can also be viewed as a restriction on the number of redundancies in a structure. In particular, in this case, bimeasurability is equivalent to the requirement that a structure have a countable number of uncountable

\footnotetext{
${ }^{6}$ We follow the definition in Kechris [14 1995]. Note, however, that this definition is equivalent to the more familiar definition, i.e., that $(\Omega, \mathcal{S}(\Omega))$ is standard Borel if it is isomorphic to a Borel subset of a Polish space. The key in showing this is Corollary 13.4 in Kechris [14, 1995].
} 
redundancies. Let us see this. Say $f: \Omega \rightarrow \Phi$ is countably uncountable if $\left\{\phi:(f)^{-1}(\{\phi\})\right.$ is uncountable\} is countable. Now, the relationship to bimeasurability:

Lemma 7.3 Fix standard Borel spaces $(\Omega, \mathcal{S}(\Omega))$ and $(\Phi, \mathcal{S}(\Phi))$. Also, fix a measurable map $f: \Omega \rightarrow \Phi$. Then $f$ is countably uncountable if and only if $f$ is bimeasurable.

Proof. Fix standard Borel spaces $(\Omega, \mathcal{S}(\Omega))$ and $(\Phi, \mathcal{S}(\Phi))$. There exists Polish spaces $(\bar{\Omega}, \mathcal{B}(\bar{\Omega}))$ and $(\bar{\Phi}, \mathcal{B}(\bar{\Phi}))$ and bijective bimeasurable maps $g: \Omega \rightarrow \bar{\Omega}$ and $k: \Phi \rightarrow \bar{\Phi}$. Define $l=k \circ f \circ\left(g^{-1}\right)$, where $f$ is as in the statement of the Lemma. Note, $l$ is a measurable map from a Polish space to a Polish space. Moreover, $(l)^{-1}(\{\bar{\phi}\})$ is uncountable if and only if $(f)^{-1}\left((k)^{-1}(\{\bar{\phi}\})\right)$ is uncountable. As such, $f$ is countably uncountable if and only if $l$ is. It follows from Purves' Theorem [19, 1966] that $l$ is bimeasurable if and only if $l$ is countably uncountable. Now note that $f=(k)^{-1} \circ l \circ g$. Since each of $g$ and $k$ are bimeasurable, $f$ is bimeasurable if and only if $l$ is bimeasurable.

Now the claim is:

Lemma 7.4 Let $(\Theta, \mathcal{S}(\Theta))$ be standard Borel and fix a $(\Theta, \mathcal{S}(\Theta))$-based standard Borel structure $\mathcal{T}$. Then $\delta^{a}$ and $\delta^{b}$ are countably uncountable if and only if they are bimeasurable.

Lemma 7.4 says the following: Fix a standard Borel parameter space and a type structure $\mathcal{T}$ that is also standard Borel. Then, $\mathcal{T}$ is bimeasurable if and only if there are (at most) a countable number of uncountable redundancies. Thus, for the standard Borel case, we have that bimeasurability is a further restriction on redundancies. To prove this result, we need to make use of two further properties of standard Borel spaces.

Property 7.1 A (finite or countable) product of standard Borel spaces is standard Borel.

Property 7.2 If $(\Omega, \mathcal{S}(\Omega))$ is standard Borel, then $(\Delta(\Omega), \mathcal{M}(\Delta(\Omega)))$ is standard Borel.

Property 7.1 is straight-forward. (See Kechris [14, 1995; page 75].) Refer to Kechris [14, 1995; page 113] for Property 7.2. (It follows from the fact that, for a Polish space $(\Omega, \mathcal{B}(\Omega)$ ), $\mathcal{M}(\Delta(\Omega))=\mathcal{B}(\Delta(\Omega))$.) Now, for the application of these properties. We will be interested in a parameter space $(\Theta, \mathcal{S}(\Theta))$ that is standard Borel. In this case, we will have that each $\left(Z_{m}, \mathcal{S}\left(Z_{m}\right)\right)$ and $\left(\Delta\left(Z_{m}\right), \mathcal{M}\left(\Delta\left(Z_{m}\right)\right)\right)$ are standard Borel, and so Lemma 7.4 follows.

Proof of Lemma 7.4. Fix a standard Borel space $(\Theta, \mathcal{S}(\Theta))$. A standard induction argument gives that, for each $m$, the sets $\left(Z_{m}, \mathcal{S}\left(Z_{m}\right)\right)$ and $\left(\Delta\left(Z_{m}\right), \mathcal{M}\left(\Delta\left(Z_{m}\right)\right)\right)$ are standard Borel. (Apply Properties $7.1,7.2$. $) \quad$ Again applying Property 7.1. $\left(\prod_{m=1}^{\infty} \Delta\left(Z_{m}\right), \mathcal{M}\left(\prod_{m=1}^{\infty} \Delta\left(Z_{m}\right)\right)\right)$ is standard Borel. As such, $\delta^{a}$ and $\delta^{b}$ are maps from a standard Borel space to a standard Borel space. The result then follows from Lemma 7.3 ,

Notice, if $\mathcal{T}$ is standard Borel, then $\mathcal{S}\left(T^{a}\right)$ and $\mathcal{S}\left(T^{b}\right)$ contain all the singletons. As such, measurable non-redundancy is equivalent to non-redundancy. So, in this case, strong measurability 
implies that the structure $\mathcal{T}$ is non-redundant, and so can be stated as a condition that depends on the type structure alone. (See Lemmata 7.1 7.2.) Conversely, if both the parameter set and $\mathcal{T}$ are standard Borel, then non-redundancy implies strong measurability. (See Lemmata 7.1.7.4.) Thus, we have the following corollary.

Corollary 7.1 Fix a parameter space $(\Theta, \mathcal{S}(\Theta))$ that is standard Borel, and a $(\Theta, \mathcal{S}(\Theta))$-based standard Borel structure $\mathcal{T}$. Then, $\mathcal{T}$ is strongly measurable if and only if $\mathcal{T}$ is non-redundant (i.e., if and only if $\mathcal{F}\left(T^{a}\right)$ and $\mathcal{F}\left(T^{b}\right)$ separate points $)$.

Let us sum up.

Corollary 7.2 Fix two $(\Theta, \mathcal{S}(\Theta))$-based structures $\mathcal{T}$ and $\mathcal{T}_{*}$. Suppose one of the following conditions obtain:

(i) $\mathcal{T}_{*}$ is strongly measurable,

(ii) $\mathcal{T}_{*}$ is measurably non-redundant and bimeasurable, or

(iii) $(\Theta, \mathcal{S}(\Theta))$ is standard Borel, $\mathcal{T}_{*}$ is standard Borel, and $\mathcal{T}_{*}$ is non-redundant.

Then, $\left(h^{a}, h^{b}\right)$ is a hierarchy morphism from $\mathcal{T}$ to $\mathcal{T}_{*}$ if and only if it is also a type morphism.

Corollary 7.2 states sufficient conditions for the hierarchy ad type morphism concepts to coincide. Obviously, condition (iii) implies condition (ii), which, in turn, implies condition (i) Condition (iii) can be stated in terms of the type structure alone, by way of Lemma 7.2 .

\section{An Alternate Approach}

The main result here states an equivalence between hierarchy and type morphisms, for the case of strongly measurable structures. There is an alternate approach to showing an equivalence. In particular, the starting point is a non-redundant $\left(\Theta, \mathcal{S}(\Theta)\right.$ )-based structure structure $\mathcal{T}_{*}$. Fix another $\left(\Theta, \mathcal{S}(\Theta)\right.$ )-based structure structure $\mathcal{T}$ and note that, by non-redundancy of $\mathcal{T}_{*}$, there is at most one hierarchy morphism from $\mathcal{T}$ to $\mathcal{I}_{*}$. As such, if it can be shown that there is some type morphism from $\mathcal{T}$ to $\mathcal{T}_{*}$, then, by Proposition 3.1, the type morphism is also a hierarchy morphism and, indeed, the unique hierarchy morphism. As such, if it can be shown that there is some type morphism from $\mathcal{T}$ to the non-redundant $\mathcal{T}_{*}$, then the concepts of hierarchy and type morphisms must coincide.

What is the relationship between the approach in Section 5 and this alternate approach? Section 5 looks at strongly measurable structures and there is a close connection between such structures and non-redundant structures. In particular, if $\mathcal{I}_{*}$ is strongly measurable and singletons are measurable-i.e., for each $t_{*}^{a} \in T_{*}^{a}\left(\right.$ resp. $\left.t_{*}^{b} \in T_{*}^{b}\right),\left\{t_{*}^{a}\right\} \in \mathcal{S}\left(T_{*}^{a}\right)$ (resp. $\left\{t_{*}^{b}\right\} \in \mathcal{S}\left(T_{*}^{b}\right)$ ) - then $\mathcal{T}_{*}$ 
is non-redundant. (See Lemma 7.1/(i)]) So, in a case of particular interest, strong measurability implies non-redundancy.

Thus, there is a connection between strong measurability and the alternate approach. But, despite this connection, it is important to note that their 'starting-points' are distinct. Under the alternate approach, we begin by verifying that there exists a type morphism from one structure to another. Under Theorem 5.1. we don't have to verify this. Instead, we verify strong measurability (e.g., by verifying the standard Borel and non-redundancy conditions). Then, we get that any hierarchy morphism is a type morphism. So, we get a type morphism as an output. That is, if there is a hierarchy morphism from one structure to another, we get - as an output - that there is also a type morphism. As such, to show that there is a type morphism from $\mathcal{T}$ to $\mathcal{T}_{*}$ it suffices to show that $\mathcal{T}_{*}$ is strongly measurable, $\delta^{a}\left(T^{a}\right) \subseteq \delta_{*}^{a}\left(T_{*}^{a}\right)$, and $\delta^{b}\left(T^{b}\right) \subseteq \delta_{*}^{b}\left(T_{*}^{b}\right)$. (See Remark 3.1.)

In sum, here we get a canonical approach to constructing a type morphism, that begins by first finding a hierarchy morphism. In practice, applications proceed along similar lines - first finding a hierarchy morphism and then verifying that the morphism constructed is indeed a type morphism. We know of no canonical approach to constructing type morphisms that does not make reference to hierarchy morphisms.

\section{Conclusion}

Throughout, we fixed two $(\Theta, \mathcal{S}(\Theta))$-based type structures, viz. $\mathcal{T}$ and $\mathcal{T}_{*}$. We have seen that, in general, a hierarchy morphism (from $\mathcal{T}$ to $\mathcal{T}_{*}$ ) may not be a type morphism (from $\mathcal{T}$ to $\mathcal{T}_{*}$ ). In particular, a necessary and sufficient condition for every hierarchy morphism (from $\mathcal{T}$ to $\mathcal{T}_{*}$ ) to be a type morphism is that the structure $\mathcal{T}_{*}$ is strongly measurable or, equivalently, that the players' language about types is determined by the players' language about hierarchies.

How should we think about this result, with an eye toward taking these results to game theory? To answer, let's recap the example in the Introduction. There, we considered the game in Figure 1.1 plus the type structures $\mathcal{T}$ and $\mathcal{T}_{*}$ as in Example 4.2 In particular, suppose the players' type structure is $\mathcal{T}$, but the analyst instead models the players' hierarchies of beliefs using the structure $\mathcal{T}_{*}$. At the surface, this seems like an equivalent modelling choice - after all, $t^{a}$ and $t_{*}^{a}$ (resp. $t^{b}, t_{*}^{b}$, and $u_{*}^{b}$ ) induce the same hierarchies of beliefs about $\Theta$. And, indeed, there is a hierarchy morphism from $\mathcal{T}$ to $\mathcal{T}_{*}$. But, recall, by doing so, the analyst introduces a new Bayesian equilibrium - one that the players' themselves have ruled out.

Here, the analyst's structure $\mathcal{T}_{*}$ is redundant (i.e., $\delta_{*}^{b}$ is not injective) and so (by Lemma 7.1 ) not strongly measurable. One first-cut answer is that it only "makes sense" for the analyst to use a type structure that is strongly measurable - after all (per Proposition 5.1) strong measurability is the requirement that the language of types is determined by the language of hierarchies (over $\Theta$ ). Yet, the robustness literature makes an interesting point: The analyst may want to use a structure that is redundant - and, so, not strongly measurable - if the analyst thinks he misspecified the 
parameter set. Specifically, suppose the analyst thinks the parameter set may be either $\Theta$ or $\Theta \times \Sigma$, where $\Sigma$ is a payoff-irrelevant signal set. Either way, the analyst thinks the players' type structure induces hierarchies of beliefs over $\Theta$ that correspond to those in $\mathcal{T}$. Then, instead of analyzing both the $(\Theta, \mathcal{S}(\Theta))$-based structure $\mathcal{T}$ and a second $(\Theta \times \Sigma, \mathcal{S}(\Theta \times \Sigma))$-based structure, he can instead analyze a redundant $\left(\Theta, \mathcal{S}(\Theta)\right.$ )-based structure like $\mathcal{T}_{*}$ and retain the Bayesian equilibrium predictions associated with the two different structures. This was the insight behind Ely-Peski's 7 , 2006] construction, and is made explicit in Liu [15, 2009].

Of course, while there is a hierarchy morphism from $\mathcal{T}$ to $\mathcal{T}_{*}$, there is no type morphism from $\mathcal{T}$ to $\mathcal{T}_{*}$. (This "fits" with Theorem 5.1 and the fact that $\mathcal{T}_{*}$ is not strongly measurable.) So, if we are concerned with robustness to misspecifying the parameter set, then perhaps we should only ask that Bayesian equilibria are preserved under type morphisms, and not under every hierarchy morphism. But now turn to Example 4.1 and note that the analyst's structure may not be strongly measurable, even if it is non-redundant. This raises the question: Should we ask that Bayesian equilibria be preserved under these hierarchy morphisms? Or might we think that it should not be preserved under such a hierarchy morphism, for a (perhaps) different robustness consideration? More loosely, is there a robustness interpretation for any failure of strong measurability? We leave these as open questions.

Finally, consider the case where the analyst is not concerned with robustness to misspecifying the parameter set. But still the analyst may have a different concern: that the players have "ruled out" hierarchies that he himself considers possible in his structure $\mathcal{T}_{*}$. In this case, he may be concerned that the players' type structure is some structure $\mathcal{T}$, so that there is a hierarchy morphism from $\mathcal{T}$ to $\mathcal{T}_{*}$. How might the results here be used? Note, since now the analyst is not concerned with robustness to misspecifying the parameter set, presumably, per Proposition 5.1, his structure $\mathcal{T}_{*}$ should be strongly measurable. If so, per Theorem 5.1, the analyst can assume that there is a type morphism from the players' structure $\mathcal{T}$ to his structure $\mathcal{T}_{*}$. This already tells the analyst that he has not introduced any new predictions by studying the larger structure. (See Proposition 4.1 in Friedenberg-Meier [9, 2007].) But, depending on the particular game studied, there may be some equilibrium prediction of the players' structure that is not an equilibrium prediction of his structure. (Again, see [9, 2007].) So, the analyst must specifically ask whether, indeed, he did lose predictions by studying the larger structure. In the course of doing so, the analyst need not leave the purview of the type structure model — after all, his hierarchy morphism is a type morphism.

\section{References}

[1] Aliprantis, C., and K. Border, Infinite Dimensional Analysis: A Hitchhiker's Guide, Springer, 1999.

[2] Armbruster, W. and W. Böge, "Bayesian Game Theory," in Game Theory and Related Topics, edited by O. Moeschlin and D. Pallaschke, North-Holland, 1979. 
[3] Battigalli, P. and A. Friedenberg, "The Context of the Game," 2009, available at http://www.public.asu.edu/ afrieden/research.htm.

[4] Böge, W., and Th. Eisele, "On Solutions of Bayesian Games," International Journal of Game Theory, 8, 1979, 193-215.

[5] Brandenburger, A., and E. Dekel, "Hierarchies of Beliefs and Common Knowledge," Journal of Economic Theory, 59, 1993, 189-189.

[6] Brandenburger, A., and A. Friedenberg, "Intrinsic Correlation in Games," Journal of Economic Theory, 141, 2008, 28-67.

[7] Ely, J., and M. Peski, "Hierarchies of Belief and Interim Rationalizability," Theoretical Economics, 1, 2006, 19-65.

[8] Dekel, E., D. Fudenberg, and S. Morris, "Interim Correlated Rationalizability," Theoretical Economics, 2, 2007, 15-40.

[9] Friedenberg, A., and M. Meier, "The Context of the Game," 2007, available at www.public.asu.edu/ afrieden/research.htm.

[10] Friedenberg, A., "Revisiting the Epistemic Conditions for Iteratively Undominated Strategies," 2008, available at http://www.public.asu.edu/ afrieden/research.htm.

[11] Harsanyi, J., "Games of Incomplete Information Played by 'Bayesian' Players, I-III," Management Science, 14, 1967-68, 159-182, 320-334, 486-502.

[12] Heifetz, A., and D. Samet, "Topology-Free Typology of Beliefs," Journal of Economic Theory, 82, 1998, 324-341.

[13] Heifetz, A., and D. Samet, "Coherent Beliefs are not Always Types," Journal of Mathematical Economics, 32, 1999, 475-488.

[14] Kechris, A., Classical Descriptive Set Theory, Springer-Verlag, 1995.

[15] Liu, Q., "On Redundant Types and Bayesian Formulation of Incomplete Information," Journal of Economic Theory, 2009, forthcoming.

[16] Meier, M., "Finitely Additive Beliefs and Universal Type Spaces," Annals of Probability, 34, 2006, 386-422.

[17] Mertens, J-F., and S. Zamir, "Formulation of Bayesian Analysis for Games with Incomplete Information," International Journal of Game Theory, 14, 1985, 1-29.

[18] Oxtoby, John C., Measure and Category: Second Edition, Springer-Verlag, 1980. 
[19] Purves, R., "Bimeasurable Functions," Fundamenta Mathematicae, 58, 1966, 149-157.

[20] Sadzik, T., "Beliefs Revealed in Bayesian-Nash Equilibrium," 2009.

[21] Siniscalchi, M., "Epistemic Game Theory: Beliefs and Types," The New Palgrave Dictionary of Economics, Second Edition, edited by L. Blume and S.N. Durlauf, Palgrave Macmillan, 2008.

[22] Weinstein, J. and M. Yildiz, "Impact of Higher-Order Uncertainty," Games and Economic Behavior, 60, 2007, 200-212.

[23] Yeh, J., Lectures on Real Analysis, World Scientific, 2000.

[24] Yildiz, M., "Consistent Equilibrium Selection," 2009. 